\title{
THE MEAN SQUARE OF THE PRODUCT OF THE RIEMANN ZETA FUNCTION WITH DIRICHLET POLYNOMIALS
}

\author{
SANDRO BETTIN, VORRAPAN CHANDEE, AND MAKSYM RADZIWIE€
}

\begin{abstract}
Improving earlier work of Balasubramanian, Conrey and Heath-Brown BCHB85, we obtain an asymptotic formula for the mean-square of the Riemann zetafunction times an arbitrary Dirichlet polynomial of length $T^{1 / 2+\delta}$, with $\delta=0.01515 \ldots$. As an application we obtain an upper bound of the correct order of magnitude for the third moment of the Riemann zeta-function. We also refine previous work of Deshouillers and Iwaniec DI84, obtaining asymptotic estimates in place of bounds. Using the work of Watt Wat95, we compute the mean-square of the Riemann zetafunction times a Dirichlet polynomial of length going up to $T^{3 / 4}$ provided that the Dirichlet polynomial assumes a special shape. Finally, we exhibit a conjectural estimate for trilinear sums of Kloosterman fractions which implies the Lindelöf Hypothesis.
\end{abstract}

\section{INTRODUCTION}

We are interested in the mean-square of the product of the Riemann zeta-function $\zeta(s)$ with an arbitrary Dirichlet polynomial $A(s)$. More precisely, we would like to understand

$$
I=\int_{\mathbb{R}}\left|\zeta\left(\frac{1}{2}+i t\right)\right|^{2} \cdot\left|A\left(\frac{1}{2}+i t\right)\right|^{2} \phi\left(\frac{t}{T}\right) d t
$$

with $\phi(x)$ a smooth function supported in $[1,2]$ and

$$
A(s):=\sum_{n \leqslant T^{\theta}} \frac{a_{n}}{n^{s}}, \quad a_{n} \ll n^{\varepsilon}, \quad \theta<1 .
$$

Asymptotic estimates for $I$ have been used consistently to understand the distribution of values of $L$-functions, the location of their zeros, and upper and lower bounds for the size of $L$-functions. See, for example, CGG86, Con89, Rad, Sou95].

It is crucially important to allow $\theta$ to be as large as possible. For example, if we could take $\theta=1-\varepsilon$ in (1.1) then the Lindelöf Hypothesis would follow.

Balasubramanian, Conrey and Heath-Brown obtained an asymptotic formula for $I$ when $\theta<\frac{1}{2}$. For $\theta<\frac{1}{2}$ and $\phi(t)$ the indicator function of the interval [1,2], they show

Date: December 1, 2014.

2010 Mathematics Subject Classification. 11M06, 11M26.

Key words and phrases. Riemann zeta function, Twisted second moment, Kloosterman fractions, Bilinear sums, Lindelöf Hypothesis.

The third author was partially supported by NSF grant DMS-1128155. 
that

$$
I=T \sum_{d, e \leqslant T^{\theta}} \frac{a_{d} \overline{a_{e}}}{[d, e]} \cdot\left(\log \left(\frac{T(d, e)^{2}}{2 \pi d e}\right)+2 \gamma+\log 4-1\right)+o(T) .
$$

When $A(s)$ is a mollifier, they show that one can go further and take $\theta<\frac{1}{2}+\frac{1}{34}=$ $0.529411 \ldots$. Their motivation was to understand the location of the zeros of the Riemann zeta-function. Specifically they deduce that at least $38 \%$ of the complex zeros of $\zeta(s)$ are on the critical line $\Re s=\frac{1}{2}$. Improvements on the admissible length of $A(s)$ will lead to a further understanding of the zeros of $\zeta(s)$ on the critical line. (See also Con89]).

In complete generality the formula (1.2) fails when $\theta>1$. Balasubramanian, Conrey and Heath-Brown conjecture that it remains true provided that $\theta<1$. This is known as the $\theta=1$ conjecture. An important change occurs at $\theta=\frac{1}{2}$. When $\theta<\frac{1}{2}$ only the diagonal terms (in the sense of Section 3.1 below) contribute to $I$, while for $\theta>\frac{1}{2}$ there is also a contribution from the non-diagonal terms which seems difficult to manage given the generality of the Dirichlet polynomial $A(s)$. The main result of our paper consists in breaking the $\frac{1}{2}$ barrier for an arbitrary Dirichlet polynomial. In fact, we prove (1.2) for $\theta<\frac{17}{33}=\frac{1}{2}+\delta$ with $\delta=\frac{1}{66} \approx 0.01515 \ldots$.

Theorem 1. Let $I$ and $A(s)$ be as above. If $\theta<\frac{1}{2}+\delta$, with $\delta=\frac{1}{66}$ then,

$$
I=\sum_{d, e \leqslant T^{\theta}} \frac{a_{d} \overline{a_{e}}}{[d, e]} \cdot \int_{\mathbb{R}}\left(\log \left(\frac{t(d, e)^{2}}{2 \pi d e}\right)+2 \gamma\right) \phi\left(\frac{t}{T}\right) d t+O\left(T^{\frac{3}{20}+\varepsilon} N^{\frac{33}{20}}+T^{\frac{1}{3}+\varepsilon}\right),
$$

where $N:=T^{\theta}$.

We notice that the off-diagonal terms contribute to the main term roughly those $d$ and $e$ for which the logarithm in the above expression is negative.

Our main tool in the proof of Theorem 1 is an estimate for trilinear forms of Kloosterman fractions, which will appear in $[\mathrm{BC}]$. This estimate improves a result of Duke, Friedlander, Iwaniec in DFI97a, dealing with bilinear sums. The use of Theorem 2 in their paper is also enough to break the $\frac{1}{2}$ barrier, though with the smaller constant $\delta=1 / 190 \approx 0.00526$ in Theorem 1 .

If we assume a general estimate for trilinear forms of Kloosterman fractions, such as,

$$
\begin{aligned}
& S_{A, M, N}:=\sum_{a} \sum_{(m, n)=1} \sum_{a} \nu_{a} \alpha_{m} \beta_{n} \mathrm{e}\left(\frac{a \bar{m}}{n}\right) \\
& \ll_{\varepsilon}\|\alpha\|\|\beta\|\|\nu\|(M+N)^{\frac{1}{2}+r+\varepsilon} A^{t}+\|\nu\| A^{\frac{1}{2}}\left(\|\alpha\|_{\infty}\|\beta\| N^{\frac{1}{2}+\varepsilon}+\|\alpha\|\|\beta\|_{\infty} M^{\frac{1}{2}+\varepsilon}\right),
\end{aligned}
$$

where $M \leq m<2 M, N \leq n<2 N, A \leq a<2 A, A \ll(N M)^{\frac{0.5-r}{1+2 t}+\varepsilon}$, and $\|\cdot\|$ and $\|\cdot\|_{\infty}$ denote the $L_{2}$ and $L_{\infty}$ norms respectively, then the statement of Theorem 1 can be replaced as follows. 
Theorem 2. Suppose that (1.3) is true for some $r, t \geq 0$. Then

$$
I=\sum_{d, e \leqslant T^{\theta}} \frac{a_{d} \overline{a_{e}}}{[d, e]} \cdot \int_{\mathbb{R}}\left(\log \left(\frac{t(d, e)^{2}}{2 \pi d e}\right)+2 \gamma\right) \phi\left(\frac{t}{T}\right) d t+O\left(T^{\frac{1}{2}-t+\varepsilon} N^{\frac{1}{2}+r+2 t}+T^{\frac{1}{3}+\varepsilon}\right),
$$

for $\theta<\frac{1}{2}+\frac{0.5-r}{1+2(r+2 t)}$ and where $N:=T^{\theta}$.

The estimate of Duke, Friedlander, Iwaniec implies (1.3) with $r=\frac{23}{48}$ and $t=\frac{1}{2}$, while the estimate of Bettin and Chandee allows us to take $r=\frac{9}{20}$ and $t=\frac{7}{20}$. We conjecture that (1.3) holds true for all $r, t \geq 0$.

Conjecture 1. Let $A \ll(N M)^{\frac{1}{2}+\varepsilon}$. Then

$$
S_{A, M, N} \ll\|\alpha\|\|\beta\|\|\nu\|(M+N)^{\frac{1}{2}+\varepsilon}+\|\nu\| A^{\frac{1}{2}}\left(\|\alpha\|_{\infty}\|\beta\| N^{\frac{1}{2}+\varepsilon}+\|\alpha\|\|\beta\|_{\infty} M^{\frac{1}{2}+\varepsilon}\right) .
$$

This conjecture essentially states that we expect square-root cancellation in the shortest two sums, as long as the total saving does not exceed $M$ or $N$. In the Appendix we show that this is best possible, up to $\varepsilon$-powers.

Using the estimate (1.4) and Theorem 2, we obtain an asymptotic formula for $I$ valid for any $\theta<1$, and this implies the Lindelöf hypothesis. We state this as a corollary below.

Corollary 1. Suppose that Conjecture 1 holds. Then the Lindelof Hypothesis is true.

Conjecture 1 appears to be strictly stronger than the Lindelöf Hypothesis. Indeed Conjecture 1 implies (1.2) with $\theta<1$, while the Lindelöf Hypothesis only gives the cruder bound

$$
I \ll T^{1+\varepsilon} \sum_{n \leq T^{\theta}} \frac{\left|a_{n}\right|^{2}}{n} .
$$

The proof of Theorem 2, on which Corollary 1 depends, is the same as that of Theorem 1 except that we use (1.3) instead of Proposition 1. The modification will be discussed at the end of Section 3 .

Duke, Friedlander and Iwaniec apply their estimate to obtain bounds for the twisted second moment of a Dirichlet $L$-function DFI97b]. They show that,

$$
\sum_{\chi \bmod q}\left|L\left(\frac{1}{2}, \chi\right)\right|^{2} \cdot\left|D\left(\frac{1}{2}, \chi\right)\right|^{2} \ll q^{1+\varepsilon}
$$

for Dirichlet polynomials $D(s, \chi)$ with coefficients $a_{n} \ll n^{\varepsilon}$ and of length $q^{1 / 2+\delta^{\prime}}$ with some $\delta^{\prime}>0$. Our proof of Theorem 1 would not extend to give an asymptotic formula in this case, and additional input is needed.

As an application of Theorem 1 we obtain an upper bound of the correct order of magnitude for the third moment of the Riemann zeta-function.

Corollary 2. We have,

$$
\int_{T}^{2 T}\left|\zeta\left(\frac{1}{2}+i t\right)\right|^{3} d t \ll T(\log T)^{9 / 4}
$$


We further indicate in Section 6.1 how to refine this result to obtain correct upper bounds for the $2 k$-th moment, when $k$ has the form $k=1+1 / n$. Previously Corollary 2 was known only on the assumption of the Riemann Hypothesis HB81. The only sharp unconditional upper bounds that were previously known are for the classic cases $k=0,1,2$ and for $k=1 / n$, due to Heath-Brown [HB81.

With further applications in mind we investigate how much $\theta$ can be increased when the Dirichlet polynomial $A(s)$ is specialized.

1.1. Products of two Dirichlet polynomials. When $A(s)$ can be written as a product of two Dirichlet polynomials $B(s) C(s)$, one can appeal to stronger estimates for sums of Kloosterman sums due to Deshouillers and Iwaniec. In [DI84], Deshouillers and Iwaniec consider the product of $\zeta(s)$ with two Dirichlet polynomials,

$$
J=\int_{\mathbb{R}}\left|\zeta\left(\frac{1}{2}+i t\right)\right|^{2} \cdot\left|A\left(\frac{1}{2}+i t\right)\right|^{2} \cdot\left|B\left(\frac{1}{2}+i t\right)\right|^{2} d t
$$

with

$$
A(s):=\sum_{n \leqslant N} \frac{\alpha_{n}}{n^{s}}, \quad B(s):=\sum_{k \leqslant K} \frac{\beta_{k}}{k^{s}}, \quad \text { where } \alpha_{n} \ll n^{\varepsilon}, \beta_{k} \ll k^{\varepsilon} .
$$

They show that if $N \geq K$, then

$$
J \ll T^{\varepsilon} \cdot\left(T+T^{1 / 2} N^{3 / 4} K+T^{1 / 2} N K^{1 / 2}+N^{7 / 4} K^{3 / 2}\right) .
$$

Their proof depends on estimates for incomplete Kloosterman sums as developed in [DI84. Proceeding similarly as in the proof of Theorem 1, and using Deshouillers and Iwaniec's estimate, we refine their bound to an asymptotic estimate.

Theorem 3. Let $J, A(s)$ and $B(s)$ be as defined in (1.5) and (1.6), and let $N \geqslant K$. Then,

$$
\begin{aligned}
J= & \sum_{d, e \leqslant N K} \frac{a_{d} \overline{a_{e}}}{[d, e]} \cdot \int_{\mathbb{R}}\left(\log \left(\frac{t(d, e)^{2}}{2 \pi d e}\right)+2 \gamma\right) \phi\left(\frac{t}{T}\right) d t \\
& +O\left(T^{\varepsilon} \cdot\left(T^{1 / 2} N^{3 / 4} K+T^{1 / 2} N K^{1 / 2}+N^{7 / 4} K^{3 / 2}\right)\right),
\end{aligned}
$$

with $a_{d}:=\sum_{n k=d} \alpha_{n} \beta_{k}$.

When the length of $N$ and $K$ is chosen suitably, Theorem 3 allows us to take $\theta<\frac{1}{2}+\frac{1}{10}$.

1.2. Specializing one of the Dirichlet polynomials. A specific case of interest is $A(s) B(s)$ with $A(s)$ of length $N=\sqrt{T}$ and smooth coefficients, and $B(s)$ arbitrary and as long as possible. One can think of such estimates as estimates for the twisted fourth moment of the Riemann zeta-function. In this case we can go further by combining the trilinear sums estimate used to prove Theorem 1 with Watt's strengthening [Wat95] of the groundbreaking work of Deshouillers-Iwaniec on estimates for sums of Kloosterman sums [DI83]. 
Theorem 4. Let $J, A(s)$ and $B(s)$ be as defined in (1.5) and (1.6). Let $N \ll T^{\frac{1}{2}+\varepsilon}$ for all $\varepsilon>0$ and assume that $\alpha_{n}=\psi(n)$ with $\psi(x)$ a smooth function such that $\psi^{(j)}(x) \ll_{j} x^{-j}$ for all $j>0$. Let $K \ll T^{\frac{1}{4}}$ and $\beta_{k} \ll k^{\varepsilon}$ for all $\varepsilon>0$. Moreover assume $\alpha_{n}$ is supported on $\left[N T^{-\xi_{1}}, 2 N\right]$ and $\beta_{k}$ is supported on $\left[K T^{-\xi_{2}}, 2 K\right]$, where $0 \leq \xi_{1} \leq \frac{1}{5}, 0 \leq \xi_{2} \leq \frac{1}{16}$. Then,

$$
\begin{aligned}
J= & \sum_{d, e \leqslant N K} \frac{a_{d} \overline{a_{e}}}{[d, e]} \cdot \int_{\mathbb{R}}\left(\log \left(\frac{t(d, e)^{2}}{2 \pi d e}\right)+2 \gamma\right) \phi\left(\frac{t}{T}\right) d t+ \\
& +O\left(T^{\frac{1}{2}+\varepsilon} K^{2}+K N^{\frac{3}{4}} T^{\frac{3}{8}+\varepsilon}+T^{\frac{39}{40}+\frac{1}{8} \xi_{1}+\frac{2}{5} \xi_{2}+\varepsilon}\right),
\end{aligned}
$$

where $a_{d}=\sum_{n k=d} \alpha_{n} \beta_{k}$.

Remark. Theorem 4 yields an asymptotic formula for $5 \xi_{1}+16 \xi_{2}<1$ (and $N \ll T^{\frac{1}{2}}$, $\left.K \ll T^{\frac{1}{2}-\varepsilon}\right)$. We remark that this range could be enlarged with a little more work.

We notice that Theorem 4 allows us to take $\theta<\frac{3}{4}$ for Dirichlet polynomials of the form $A(s) B(s)$ with $A(s)$ pretending to be $\zeta(s)$ and $B(s)$ of length up to $T^{1 / 4-\varepsilon}$. Thus, following the work of Radziwiłł Rad12, Theorem 4 could be applied to give a sharp upper bound for the $2 k$-th moment of the Riemann zeta function for $2 k<$ 5 , conditionally on the Riemann hypothesis (however, we remark that this has been recently proven for all $k \geq 0$ by Harper [Har]). It would be interesting to investigate if Theorem 4 has other applications, for example to the study of large gaps between the zeros of the Riemann zeta-function (see [Bre]).

Theorem 4 refines upon Watt's result, who uses his Kloosterman sum estimate to give (essentially) an upper bound of the form $J \ll T^{1+\varepsilon}+T^{1 / 2+\varepsilon} K^{2}$, for $a_{n}, b_{n}$ supported on dyadic intervals. Theorem 4 should also be compared with the asymptotic formula for the twisted fourth moment of Hughes and Young [HY10. Their result allows to get an asymptotic formula for the second moment of $\zeta^{2}(s) B(s)$ with $B(s)$ of length up to $T^{1 / 11-\varepsilon}$.

\section{ACKNOWLEDGMENTS}

We are very grateful to Brian Conrey for suggesting to us the problem of breaking the $\frac{1}{2}$ barrier in Theorem 1 and to Micah B. Milinovich and Nathan Ng for pointing out the paper of Duke, Friedlander, Iwaniec [DFI97a]. We also wish to thank the referee for a very careful reading of the paper and for indicating several inaccuracies and mistakes.

\section{Estimates for sums of Kloosterman sums}

Remark. Throughout the paper, we use the common convention in analytic number theory that $\varepsilon$ denotes an arbitrarily small positive quantity that may vary from line to line.

In this section, we collect the estimates for sums of Kloosterman sums that will be used to prove the theorems. 
The following Proposition is from $[\mathrm{BC}$, and we will use it when dealing with the contribution of the off-diagonal terms in Theorem 1 and 4.

Proposition 1. Let $\alpha_{m}, \beta_{n}, \nu_{a}$ be complex numbers, where $M \leq m<2 M, N \leq n<$ $2 N$, and $A \leq a<2 A$. Then for any $\varepsilon>0$, we have

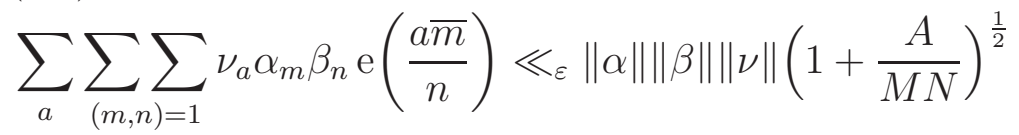

$$
\begin{aligned}
& \times\left((A M N)^{\frac{7}{20}+\varepsilon}(M+N)^{\frac{1}{4}}+(A M N)^{\frac{3}{8}+\varepsilon}(A N+A M)^{\frac{1}{8}}\right),
\end{aligned}
$$

where $\|\cdot\|$ denotes the $L_{2}$ norm.

The off-diagonal terms in Theorem 3 will be estimated using the following bound, due to Deshouillers and Iwaniec [DI84.

Proposition 2 (Deshouillers, Iwaniec). Let $L, J, U, V \geq 1$ and $|c(u, v)| \leq 1$. We then have

$$
\begin{aligned}
& \sum_{\substack { 1 \leq \ell \leq L \\
\begin{subarray}{c}{1 \leq j \leq J \\
(\ell, \varrho j)=1{ 1 \leq \ell \leq L \\
\begin{subarray} { c } { 1 \leq j \leq J \\
( \ell , \varrho j ) = 1 } }\end{subarray}}\left|\sum_{\substack { 1 \leq u \leq U \\
\begin{subarray}{c}{1 \leq v \leq V \\
(v, \ell)=1{ 1 \leq u \leq U \\
\begin{subarray} { c } { 1 \leq v \leq V \\
( v , \ell ) = 1 } }\end{subarray}} c(u, v) \mathrm{e}\left(u \frac{\overline{\varrho v j}}{\ell}\right)\right| \\
& \ll(L J U V)^{1 / 2+\varepsilon}\left\{(L J)^{1 / 2}+(U+V)^{1 / 4}\left[L J(U+\varrho V)\left(L+\varrho V^{2}\right)+\varrho U V^{2} J^{2}\right]^{1 / 4}\right\} .
\end{aligned}
$$

Finally, to estimate the off-diagonal terms in Theorem 4, we will use the following Proposition, which can be derived easily from Proposition 4.1 of Watt [Wat95.

Proposition 3 (Watt). Let $H, C, P, V, R, S \geq 1$ and $\delta \leq 1$. Assume that for some $\varepsilon>0$ we have

$$
\begin{aligned}
X & :=\left(\frac{R V S P}{H C}\right)^{\frac{1}{2}} \gg(R S P V)^{\varepsilon}, \\
(R S)^{2} & \geq \max \left(H^{2} C, \frac{S P}{V}(R S P V)^{\varepsilon}\right) .
\end{aligned}
$$

Moreover, assume that $\alpha(x), \beta(x)$ are complex valued smooth functions, supported on the intervals $[1 / 2, H]$ and $[1 / 2, C]$ respectively, such that

$$
\alpha^{(j)}(x), \beta^{(j)}(x) \ll_{j}(\delta x)^{-j}
$$

for all $j \geq 0$. Assume $a_{r}, b_{s}$ are sequences of complex numbers supported on $[R / 2, R]$, $[S / 2, S]$ respectively and are such that $a_{r} \ll r^{\varepsilon}, b_{s} \ll s^{\varepsilon}$. Finally, assume that for all $i, j \geq 0$,

$$
\frac{d^{i+j}}{d y^{i} d y^{j}} \gamma_{r, s}(x, y) \ll_{i, j} x^{-i} y^{-j}
$$


where for all $r$ and $s, \gamma_{r, s}(x, y)$ is supported on $[V / 2, V] \times[P / 2, P]$. Then

$$
\begin{aligned}
& \sum_{s \sim S,} \sum_{r \sim R} \sum \sum_{\substack{h, c, p, v,(r v, s p)=1}} \sum \alpha(h) \beta(c) \gamma_{r, s}(v, p) a_{r} b_{s} \mathrm{e}\left( \pm \frac{h c \overline{r v}}{s p}\right) \\
& \ll \delta^{-\frac{7}{2}} H C R(V+S X)\left(1+\frac{H C}{R S}\right)^{\frac{1}{2}}\left(1+\frac{P}{V R}\right)^{\frac{1}{2}}\left(1+\frac{H^{2} C P X^{2}}{V S^{3} R^{4}}\right)^{\frac{1}{4}}(H C R V P S)^{20 \varepsilon} .
\end{aligned}
$$

Proof. Firstly using smooth partitions of unity, we can assume that $\alpha(x), \beta(x)$ are supported on $[H / 2, H]$ and $[C / 2, C]$, since the bound (2.3) is weaker (and the conditions (2.2) stricter) for larger values of $H$ and $C$. Moreover, by dividing by $R^{\varepsilon}$ and $S^{\varepsilon}$ if necessary, we can assume $a_{r}, b_{s} \ll 1$.

By Poisson's formula,

$$
\begin{aligned}
\sum_{(v, s p)=1} \gamma(v, p) \mathrm{e}\left( \pm \frac{h c \overline{r v}}{s p}\right) & =\sum_{u(\bmod s p)}^{*} \mathrm{e}\left( \pm \frac{h c \overline{r u}}{s p}\right) \sum_{v \equiv u(\bmod s p)} \gamma(v, p) \\
& =\sum_{u(\bmod s p)}^{*} \mathrm{e}\left( \pm \frac{h c \overline{r u}}{s p}\right) \frac{1}{s p} \sum_{\ell} \mathrm{e}\left(-\frac{\ell u}{s p}\right) \int_{\mathbb{R}} \gamma(y, p) \mathrm{e}\left(\frac{\ell y}{s p}\right) d y \\
& =\sum_{\ell} S(h c \bar{r}, \mp \ell, s p) \int_{\mathbb{R}} \gamma(y s p, p) \mathrm{e}(\ell y) d y .
\end{aligned}
$$

If $\ell=0$, the Kloosterman sum reduces to a Ramanujan sum, and one has $S(h c \bar{r}, \mp \ell, s p) \ll$ $(h c, s p)$. Thus, the contribution to (2.3) coming from the terms $\ell=0$ is bounded by

$$
\sum_{s \sim S} \sum_{r \sim R} \sum \sum_{\substack{h, c, p \\(r, s p)=1}} \sum \alpha(h) \beta(c) a_{r} b_{s} \frac{(h c, s p)}{s p} V \ll R V H C(H C P)^{\varepsilon} .
$$

Also, integrating by parts repeatedly, we see that the terms with $\ell \geq \frac{S P}{V}(R S P V)^{\varepsilon}$ give a negligible contribution. For the remaining terms, we introduce a smooth partition of unity

$$
1=\sum_{L}^{\prime} \theta_{L}(x), \quad \forall x \geq 1
$$

where $\theta_{L}(x)$ is supported in $[L / 2,3 L]$ (with $L \ll \frac{S P}{V}(R S P V)^{\varepsilon}$ ), satisfies $\theta_{L}(x)^{j} \ll_{j} L^{-j}$ for all $j \geq 0$, and is such that $\sum_{L \leq X}^{\prime} 1 \ll \log (2+X)$ for all $X \geq 1$. Thus, we need to bound

$$
\begin{aligned}
& \sum_{s \sim S,} \sum_{r \sim R} \sum \sum_{\substack{h, c, p,(r, s p)=1}} \sum_{0<|\ell|<\frac{S P}{V}(R S P V)^{\varepsilon}} \alpha(h) \beta(c) a_{r} b_{s} S(h c \bar{r}, \mp \ell, s p) \int_{\mathbb{R}} \gamma(y s p, p) \mathrm{e}(\ell y) d y \\
& =\sum_{L}^{\prime} \int_{y \sim \frac{V}{S P}} \sum_{s \sim S, r \sim R} \sum_{\substack{h, c, p,(r, s p)=1}} \sum_{\ell \sim L} \sum_{\ell \sim L} \alpha(h) \beta(c) \omega(\ell, y) a_{r} b_{s} S(h c \bar{r}, \mp \ell, s p) f_{s}(p, y) d y
\end{aligned}
$$


where $f_{s}(p, y):=\gamma(y s p, p), \omega(\ell, y)=\theta(\ell) \mathrm{e}(\ell y)$, and $\sum^{\prime}$ denotes the sum over the partitions of unity. We remark that for $y \sim \frac{V}{S P}$, we have $\frac{d^{j}}{d p^{j}} f_{s}(p, y) \ll p^{-j}$, and that $\frac{d^{j}}{d \ell^{j}} \omega(\ell, y) \ll\left(L^{-1}+\frac{V}{S P}\right)^{j} \ll L^{-j}(R S P V)^{j \varepsilon}$. By Proposition 4.1 of Watt Wat95, the sums inside the integral are bounded by

$$
\delta^{-\frac{7}{2}}(R V L P)^{\frac{7}{2} \varepsilon} H C L(R S X)^{1+\varepsilon}\left(1+\frac{H C}{R S}\right)^{\frac{1}{2}}\left(1+\frac{L}{R S}\right)^{\frac{1}{2}}\left(1+\frac{H^{2} C L X^{2}}{(R S)^{4}}\right)^{\frac{1}{4}}
$$

and summing over $L$ and integrating over $y$ completes the proof of the proposition.

\section{The Proof of TheOrem 1}

We start by expressing $\left|\zeta\left(\frac{1}{2}+i t\right)\right|^{2}$ as a sum of length approximately $T^{1+\varepsilon}$. Let $G(w)$ be an entire function with rapid decay along vertical lines, that is $G(x+i y) \ll y^{-A}$ for any fixed $x$ and $A>0$. Suppose $G(-w)=G(w), G(0)=1, G(1 / 2)=0$. We will use the following form of the approximate functional equation for $|\zeta(s)|^{2}$.

Lemma 1 (Approximate functional equation). For $T<t<2 T$, we have

$$
\left|\zeta\left(\frac{1}{2}+i t\right)\right|^{2}=2 \sum_{m_{1}, m_{2}} \frac{1}{\left(m_{1} m_{2}\right)^{\frac{1}{2}}}\left(\frac{m_{1}}{m_{2}}\right)^{i t} W\left(\frac{2 \pi m_{1} m_{2}}{t}\right)+O\left(T^{-2 / 3}\right),
$$

where

$$
W(x):=\frac{1}{2 \pi i} \int_{(2)} x^{-w} G(w) \frac{d w}{w}
$$

and where we use the notation $\int_{(c)}$ to mean an integration up the vertical line from $c-i \infty$ to $c+i \infty$.

The proof of the lemma can be found in Lemma 3 of [LR].

Remark. Notice that $W^{(\ell)}(x) \ll_{\ell, A} \min \left(1, x^{-A}\right)$ for $x>0$ and all $\ell \in \mathbb{N}$.

The error term in Lemma 1 produces an error term bounded by $T^{\frac{1}{3}+\varepsilon}$, and thus

$$
\begin{aligned}
I & =2 \sum_{n_{1}, n_{2}, m_{1}, m_{2}} \frac{a_{n_{1}} \bar{a}_{n_{2}}}{\left(m_{1} m_{2} n_{1} n_{2}\right)^{\frac{1}{2}}} \int_{\mathbb{R}}\left(\frac{m_{1} n_{2}}{m_{2} n_{1}}\right)^{i t} W\left(\frac{2 \pi m_{1} m_{2}}{t}\right) \phi\left(\frac{t}{T}\right) d t+O\left(T^{\frac{1}{3}+\varepsilon}\right) \\
& =\mathcal{D}+\mathcal{S}+O\left(T^{\frac{1}{3}+\varepsilon}\right),
\end{aligned}
$$

where the sum is over $n_{1}, n_{2} \leq N, \mathcal{D}$ is the sum when $m_{1} n_{2}=m_{2} n_{1}$, and $\mathcal{S}$ is the sum when $m_{1} n_{2} \neq m_{2} n_{1}$.

3.1. Diagonal terms. Firstly, we consider the diagonal terms $m_{1} n_{2}=m_{2} n_{1}$. For $j=1,2$, we write $m_{j}=\ell n_{j}^{*}$, where $n_{j}^{*}=\frac{n_{j}}{\left(n_{1}, n_{2}\right)}$. The contribution of the diagonal term 
is

$$
\begin{aligned}
\mathcal{D}=2 \sum_{n_{1}, n_{2}, \ell} & \frac{a_{n_{1}} \bar{a}_{n_{2}}\left(n_{1}, n_{2}\right)}{\ell n_{1} n_{2}} \int_{\mathbb{R}} W\left(\frac{2 \pi \ell^{2} n_{1}^{*} n_{2}^{*}}{t}\right) \phi\left(\frac{t}{T}\right) d t \\
& =\frac{2}{2 \pi i} \sum_{n_{1}, n_{2}, \ell} \frac{a_{n_{1}} \bar{a}_{n_{2}}\left(n_{1}, n_{2}\right)}{\ell n_{1} n_{2}} \int_{\mathbb{R}} \int_{(2)}\left(\frac{2 \pi \ell^{2} n_{1}^{*} n_{2}^{*}}{t}\right)^{-w} G(w) \frac{d w}{w} \phi\left(\frac{t}{T}\right) d t \\
& =\frac{2}{2 \pi i} \sum_{n_{1}, n_{2}} \frac{a_{n_{1}} \bar{a}_{n_{2}}\left(n_{1}, n_{2}\right)}{n_{1} n_{2}} \int_{\mathbb{R}} \int_{(2)}\left(\frac{t}{2 \pi n_{1}^{*} n_{2}^{*}}\right)^{w} \zeta(1+2 w) G(w) \frac{d w}{w} \phi\left(\frac{t}{T}\right) d t .
\end{aligned}
$$

This term will be later combined with a contribution from the off-diagonal terms. Together, they give the main term in Theorem 1 .

3.2. Off-Diagonal terms. In this section, we consider the terms with $m_{1} n_{2} \neq m_{2} n_{1}$. We write $m_{1} n_{2}-m_{2} n_{1}=\Delta$.

Since $W(x) \ll x^{-A}$ when $x \gg 1$, we can truncate the sum over $m_{1}, m_{2}$ to when $m_{1} m_{2} \leq T^{1+\varepsilon}$. We introduce a smooth partition of unity

$$
1=\sum_{M}^{\prime} F_{M}(x), \quad T^{-100} \leq x \leq T^{1+\varepsilon}
$$

where $F_{M}(x)$ is smooth, supported in $[M / 2,3 M]$, and it satisfies $F_{M}^{(j)}(x) \ll_{j} \frac{1}{M^{j}}$ for all $j \geq 0$. Moreover we can choose a partition of unity which satisfies $\sum_{M}^{\prime} 1 \ll \log (2+T)$. Therefore

$$
\begin{aligned}
\mathcal{S}= & 2 \sum_{N_{1}}^{\prime} \sum_{N_{2}}^{\prime} \sum_{M}^{\prime} \sum_{\Delta \neq 0} \sum_{\substack{n_{1}, n_{2}, m_{1}, m_{2} \\
m_{1} n_{2}-m_{2} n_{1}=\Delta}} \frac{a_{n_{1}} \bar{a}_{n_{2}}}{\left(m_{1} m_{2} n_{1} n_{2}\right)^{\frac{1}{2}}} \\
& \times\left(\int_{\mathbb{R}}\left(1+\frac{\Delta}{m_{2} n_{1}}\right)^{i t} W\left(\frac{2 \pi m_{1} m_{2}}{t}\right) \phi\left(\frac{t}{T}\right) d t\right) F_{N_{1}}\left(n_{1}\right) F_{N_{2}}\left(n_{2}\right) F_{M}\left(m_{2}\right)+O(1),
\end{aligned}
$$

where $N_{1}, N_{2} \leq N$ and $M \leq T^{1+\varepsilon}$.

Next we show that the terms with $|\Delta|>D, D:=\frac{M N_{1}}{T^{1-\varepsilon}}$, give a negligible contribution. In fact,

$$
\frac{d^{\ell}}{d t^{\ell}} W\left(\frac{2 \pi m_{1} m_{2}}{t}\right) \ll_{\ell, A} \frac{1}{t^{\ell}} \min \left(1,\left(\frac{2 \pi m_{1} m_{2}}{t}\right)^{-A}\right)
$$


whence, integrating by part $\ell$ times, we have

$$
\begin{gathered}
\sum_{N_{1}, N_{2}, M}^{\prime} \sum_{|\Delta|>D} \sum_{n_{1}, m_{2}} \sum_{\substack{n_{2}, m_{1} \\
m_{1} n_{2}-m_{2} n_{1}=\Delta}} \frac{a_{n_{1}} \bar{a}_{n_{2}}}{\left(m_{1} m_{2} n_{1} n_{2}\right)^{\frac{1}{2}}}\left(\int_{\mathbb{R}}\left(1+\frac{\Delta}{m_{2} n_{1}}\right)^{i t} W\left(\frac{2 \pi m_{1} m_{2}}{t}\right) \phi\left(\frac{t}{T}\right) d t\right) \\
\quad \times F_{N_{1}}\left(n_{1}\right) F_{N_{2}}\left(n_{2}\right) F_{M}\left(m_{2}\right) \\
\ll \sum_{N_{1}, M}^{\prime} \sum_{|\Delta|>D} \sum_{n_{1} \sim N_{1}, m_{2} \sim M} \sum_{\substack{m_{1} \leq T_{1+\varepsilon}^{1+\varepsilon} \\
n_{2} \leq N, m_{1} n_{2}-m_{2} n_{1}=\Delta}} \frac{T^{-\ell+1+\varepsilon}}{\left(m_{1} m_{2} n_{1} n_{2}\right)^{\frac{1}{2}}}\left|\log \left(1+\frac{\Delta}{m_{2} n_{1}}\right)\right|^{-\ell} \\
\quad \frac{1}{\sqrt{n_{1} m_{2}}} \sum_{\substack{m_{1} \leq T^{1+\varepsilon} \\
n_{2} \leq N, m_{1}-m_{2} n_{1}=\Delta}} \frac{1}{\sqrt{n_{2} m_{1}}} T^{-\ell+1+\varepsilon} \cdot\left(\frac{m_{2} n_{1}}{|\Delta|}\right)^{\ell} \ll_{A, \varepsilon} T^{-A},
\end{gathered}
$$

where $\ell$ is large enough.

Now, if $|\Delta|<D$, then $\frac{\Delta}{m_{2} n_{1}} \ll \frac{1}{T^{1-\varepsilon}}$, and

$$
m_{1}=\frac{m_{2} n_{1}+\Delta}{n_{2}}=m_{2} \frac{n_{1}}{n_{2}}\left(1+\frac{\Delta}{m_{2} n_{1}}\right) .
$$

Hence for $T<t<2 T$,

$$
\begin{gathered}
\frac{1}{m_{1}}=\frac{n_{2}}{m_{2} n_{1}}\left(1-\frac{\Delta}{m_{2} n_{1}}+O\left(\frac{1}{T^{2-\varepsilon}}\right)\right) \\
\left(1+\frac{\Delta}{m_{2} n_{1}}\right)^{i t}=e^{i t \log \left(1+\frac{\Delta}{m_{2} n_{1}}\right)}=e^{i t \frac{\Delta}{m_{2} n_{1}}}\left(1-\frac{i t \Delta^{2}}{2 m_{2}^{2} n_{1}^{2}}+O\left(\frac{1}{T^{2-\varepsilon}}\right)\right),
\end{gathered}
$$

and

$$
W\left(\frac{2 \pi m_{1} m_{2}}{t}\right)=W\left(\frac{2 \pi m_{2}^{2} n_{1}}{t n_{2}}\right)+\frac{2 \pi m_{2} \Delta}{t n_{2}} W^{\prime}\left(\frac{2 \pi m_{2}^{2} n_{1}}{t n_{2}}\right)+O\left(\frac{1}{T^{2-\varepsilon}}\right)
$$

Since $m_{1} m_{2} \leq T^{1+\varepsilon}$, we have $m_{2}\left(m_{2} n_{1}+\Delta\right) \leq n_{2} T^{1+\varepsilon}$. Hence $M \ll T^{1 / 2+\varepsilon} \sqrt{\frac{N_{2}}{N_{1}}}$, and the error term from using the above approximations in (3.3) is

$$
\begin{aligned}
& \ll \frac{T}{T^{2-\varepsilon}} \sum_{N_{1}}^{\prime} \sum_{N_{2}}^{\prime} \sum_{M \ll T^{1 / 2+\varepsilon}}^{\prime} \sum_{\sqrt{\frac{N_{2}}{N_{1}}}} \sum_{0<|\Delta| \leq D} \sum_{\substack{n_{1} \sim N_{1} \\
n_{2} \sim N_{2}}} \frac{1}{m_{2} \sim M} \\
& \ll \sum_{N_{1}}^{\prime} \sum_{N_{2}}^{\prime} \sum_{M \ll T^{1 / 2+\varepsilon} \sqrt{N_{2} / N_{1}}}^{\prime} \frac{M N_{1} N_{2}}{T^{2-\varepsilon}} \ll \frac{\sqrt{T} N_{2}^{3 / 2} N_{1}^{1 / 2}}{T^{2-\varepsilon}} \ll \frac{N^{2}}{T^{3 / 2-\varepsilon}},
\end{aligned}
$$

using that $D=M N_{1} / T^{1-\varepsilon}$ and that $M \ll T^{1 / 2+\varepsilon} \sqrt{\frac{N_{2}}{N_{1}}}$. Thus, we have

$$
\mathcal{S}=\mathcal{A}+\mathcal{E}+O\left(1+\frac{N^{2}}{T^{3 / 2-\varepsilon}}\right)
$$


where

$$
\begin{aligned}
\mathcal{A}= & 2 \sum_{N_{1}}^{\prime} \sum_{N_{2}}^{\prime} \sum_{\substack{M \leq T^{1 / 2+\varepsilon} \\
\sqrt{\frac{N_{2}}{N_{1}}}}}^{\prime} \sum_{0<|\Delta| \leq D} \sum_{n_{1}, n_{2}} \sum_{\substack { n_{1} m_{2} \equiv-\Delta\left(\bmod n_{2}\right) \\
\begin{subarray}{c}{m_{2}>0{ n _ { 1 } m _ { 2 } \equiv - \Delta ( \operatorname { m o d } n _ { 2 } ) \\
\begin{subarray} { c } { m _ { 2 } > 0 } }\end{subarray}} \frac{a_{n_{1}} \bar{a}_{n_{2}}}{m_{2} n_{1}} \times \\
& \times\left(\int_{\mathbb{R}} \mathrm{e}\left(\frac{\Delta t}{2 \pi m_{2} n_{1}}\right) W\left(\frac{2 \pi m_{2}^{2} n_{1}}{t n_{2}}\right) \phi\left(\frac{t}{T}\right) d t\right) F_{M}\left(m_{2}\right) F_{N_{1}}\left(n_{1}\right) F_{N_{2}}\left(n_{2}\right),
\end{aligned}
$$

and

$$
\begin{aligned}
\mathcal{E}= & 2 \sum_{N_{1}}^{\prime} \sum_{N_{2}}^{\prime} \sum_{M \leq T^{1 / 2+\varepsilon}}^{\prime} \sum_{\sqrt{\frac{N_{2}}{N_{1}}}} \sum_{0<|\Delta| \leq D} \sum_{n_{1}, n_{2}} \sum_{\substack{n_{1} m_{2}=-\Delta\left(\bmod n_{2}\right) \\
m_{2}>0}} \frac{a_{n_{1}} \bar{a}_{n_{2}}}{m_{2} n_{1}} F_{M}\left(m_{2}\right) F_{N_{1}}\left(n_{1}\right) F_{N_{2}}\left(n_{2}\right) \times \\
& \times \int_{\mathbb{R}} \mathrm{e}\left(\frac{\Delta t}{2 \pi m_{2} n_{1}}\right)\left[W\left(\frac{2 \pi m_{2}^{2} n_{1}}{t n_{2}}\right)\left(-\frac{\Delta}{2 m_{2} n_{1}}-\frac{i t \Delta^{2}}{2 m_{2}^{2} n_{1}^{2}}\right)+\frac{2 \pi m_{2} \Delta}{t n_{2}} W^{\prime}\left(\frac{2 \pi m_{2}^{2} n_{1}}{t n_{2}}\right)\right] \phi\left(\frac{t}{T}\right) d t,
\end{aligned}
$$

since the rest of the terms arising from the above approximations also give a contribution which is $O\left(N^{2} T^{-3 / 2+\varepsilon}\right)$.

First, we consider $\mathcal{A}$. Giving an eligible bound for $\mathcal{E}$ is easy and we will do it in the next section.

Extracting the common divisor $d$ of $n_{1}$ and $n_{2}$, we re-write the sum (3.4) as

$$
\mathcal{A}=2 \sum_{d \leq N} \frac{1}{d} \sum_{N_{1}, N_{2} \leq N}^{\prime} \sum_{M \leq T^{1 / 2+\varepsilon}}^{\prime} \sum_{\sqrt{\frac{N_{2}}{N_{1}}}} \sum_{0<|\Delta| \leq \frac{D}{d}} \sum_{\substack{n_{1}, n_{2} \\\left(n_{1}, n_{2}\right)=1}} a_{d n_{1}} \bar{a}_{d n_{2}} F_{N_{1}}\left(d n_{1}\right) F_{N_{2}}\left(d n_{2}\right) \mathcal{A}_{M, N_{i}}\left(n_{1}, n_{2}, \Delta\right),
$$

where

$$
\mathcal{A}_{M, N_{i}}\left(n_{1}, n_{2}, \Delta\right)=\sum_{m_{2} \equiv-\overline{n_{1}} \Delta\left(\bmod n_{2}\right)} \frac{F_{M}\left(m_{2}\right)}{m_{2} n_{1}}\left(\int_{\mathbb{R}} \mathrm{e}\left(\frac{\Delta t}{2 \pi m_{2} n_{1}}\right) W\left(\frac{2 \pi m_{2}^{2} n_{1}}{t n_{2}}\right) \phi\left(\frac{t}{T}\right) d t\right) .
$$

By Poisson summation formula,

$$
\begin{aligned}
\mathcal{A}_{M, N_{i}}\left(n_{1}, n_{2}, \Delta\right)= & \frac{1}{n_{1} n_{2}} \sum_{h \in \mathbb{Z}} \mathrm{e}\left(-\frac{h \bar{n}_{1} \Delta}{n_{2}}\right) \int_{0}^{\infty} \mathrm{e}\left(-\frac{h x}{n_{2}}\right) \frac{F_{M}(x)}{x} \times \\
& \times \int_{\mathbb{R}} \mathrm{e}\left(\frac{\Delta t}{2 \pi x n_{1}}\right) W\left(\frac{2 \pi x^{2} n_{1}}{t n_{2}}\right) \phi\left(\frac{t}{T}\right) d t d x .
\end{aligned}
$$

After the change of variable $x \rightarrow \frac{x}{n_{1}}$, this becomes

$$
\mathcal{A}_{M, N_{i}}\left(n_{1}, n_{2}, \Delta\right)=\frac{1}{n_{1} n_{2}} \sum_{h \in \mathbb{Z}} \widetilde{\mathcal{A}}_{M, N_{i}}\left(h, n_{1}, n_{2}, \Delta\right) \mathrm{e}\left(-\frac{h \bar{n}_{1} \Delta}{n_{2}}\right),
$$

where

$$
\widetilde{\mathcal{A}}_{M, N_{i}}\left(h, n_{1}, n_{2}, \Delta\right)=\int_{0}^{\infty} \mathrm{e}\left(-\frac{h x}{n_{1} n_{2}}\right) \frac{F_{M}\left(\frac{x}{n_{1}}\right)}{x} \int_{\mathbb{R}} \mathrm{e}\left(\frac{\Delta t}{2 \pi x}\right) W\left(\frac{2 \pi x^{2}}{n_{1} n_{2} t}\right) \phi\left(\frac{t}{T}\right) d t d x .
$$

To understand the contribution of $\widetilde{\mathcal{A}}_{M, N_{i}}\left(h, n_{1}, n_{2}, \Delta\right)$, we consider the following three cases. 
Case 1: $h=0$. The contribution to $\mathcal{A}$ from $h=0$ is

$$
\begin{aligned}
\mathcal{A}_{0}=2 \sum_{d \leq N} \sum_{N_{1} \leq N}^{\prime} & \sum_{N_{2} \leq N}^{\prime} \sum_{M \leq T^{1 / 2+\varepsilon}}^{\prime} \sum_{\sqrt{\frac{N_{2}}{N_{1}}}} \sum_{0<|\Delta| \leq \frac{D}{d}} \frac{a_{n_{1}, n_{2}} \bar{a}_{d n_{2}} F_{\left.N_{1}, n_{2}\right)=1}\left(d n_{1}\right) F_{N_{2}}\left(d n_{2}\right)}{d n_{1} n_{2}} \\
& \times \int_{\mathbb{R}} \int_{0}^{\infty} F_{M}\left(\frac{x}{n_{1}}\right) \mathrm{e}\left(\frac{\Delta t}{2 \pi x}\right) W\left(\frac{2 \pi x^{2}}{n_{1} n_{2} t}\right) \frac{d x}{x} \phi\left(\frac{t}{T}\right) d t .
\end{aligned}
$$

Now, we can extend the sum over $\Delta$ to $\Delta \in \mathbb{Z} \backslash\{0\}$, since it can be shown as before that the terms $|\Delta| \geq D / d$ give a negligible contribution. Making the change of variables $y=t / x$ and integrating by parts twice we see that the second line of (3.6) is equal to

$$
\begin{aligned}
& -\frac{1}{\Delta^{2}} \int_{\mathbb{R}} \int_{0}^{\infty} \mathrm{e}\left(\frac{\Delta y}{2 \pi}\right) \frac{d^{2}}{d y^{2}}\left(F_{M}\left(\frac{t}{n_{1} y}\right) W\left(\frac{2 \pi t}{n_{1} n_{2} y^{2}}\right) \frac{1}{y}\right) d y \phi\left(\frac{t}{T}\right) d t \\
& =-\frac{1}{\Delta^{2}} \int_{\mathbb{R}} \int_{R\left(t, n_{1}\right)} \mathrm{e}\left(\frac{\Delta y}{2 \pi}\right) \frac{d^{2}}{d y^{2}}\left(F_{M}\left(\frac{t}{n_{1} y}\right) W\left(\frac{2 \pi t}{n_{1} n_{2} y^{2}}\right) \frac{1}{y}\right) d y \phi\left(\frac{t}{T}\right) d t+O\left(\frac{1}{\Delta^{2}}\right),
\end{aligned}
$$

where $R\left(t, n_{1}\right)=\left\{y \mid T^{-100}<\frac{t}{n_{1} y}<T^{1 / 2+\varepsilon} \sqrt{\frac{N_{2}}{N_{1}}}\right\}$ and where we estimated trivially the part of the integral over $y$ with $y \in \mathbb{R}_{>0} \backslash R\left(t, n_{1}\right)$, using the properties of $W$ and $F_{M}$ (and $n_{1} \ll T$ ). Thus, summing over $M$ we have

$$
\begin{aligned}
& \sum_{M}^{\prime} \int_{\mathbb{R}} \int_{0}^{\infty} F_{M}\left(\frac{x}{n_{1}}\right) \mathrm{e}\left(\frac{\Delta t}{2 \pi x}\right) W\left(\frac{2 \pi x^{2}}{n_{1} n_{2} t}\right) \frac{d x}{x} \phi\left(\frac{t}{T}\right) d t= \\
& \quad=-\frac{1}{\Delta^{2}} \int_{\mathbb{R}} \int_{R\left(t, n_{1}\right)} \mathrm{e}\left(\frac{\Delta y}{2 \pi}\right) \frac{d^{2}}{d y^{2}}\left(W\left(\frac{2 \pi t}{n_{1} n_{2} y^{2}}\right) \frac{1}{y}\right) d y \phi\left(\frac{t}{T}\right) d t+O\left(\frac{\log (2+T)}{\Delta^{2}}\right) \\
& \quad=-\frac{1}{\Delta^{2}} \int_{\mathbb{R}} \int_{0}^{\infty} \mathrm{e}\left(\frac{\Delta y}{2 \pi}\right) \frac{d^{2}}{d y^{2}}\left(W\left(\frac{2 \pi t}{n_{1} n_{2} y^{2}}\right) \frac{1}{y}\right) d y \phi\left(\frac{t}{T}\right) d t+O\left(\frac{\log (2+T)}{\Delta^{2}}\right) .
\end{aligned}
$$

Therefore, summing over $N_{1}, N_{2}$, we have

$$
\begin{aligned}
\mathcal{A}_{0}= & -2 \sum_{d \leq N} \sum_{\substack { \Delta \mid \neq 0 \\
\begin{subarray}{c}{n_{1}, n_{2} \leq \frac{N}{d} \\
\left(n_{1}, n_{2}\right)=1{ \Delta | \neq 0 \\
\begin{subarray} { c } { n _ { 1 } , n _ { 2 } \leq \frac { N } { d } \\
( n _ { 1 } , n _ { 2 } ) = 1 } }\end{subarray}} \frac{a_{d n_{1}} \bar{a}_{d n_{2}}}{d n_{1} n_{2} \Delta^{2}} \int_{\mathbb{R}} \int_{0}^{\infty} \mathrm{e}\left(\frac{\Delta y}{2 \pi}\right) \frac{d^{2}}{d y^{2}}\left(W\left(\frac{2 \pi t}{n_{1} n_{2} y^{2}}\right) \frac{1}{y}\right) d y \phi\left(\frac{t}{T}\right) d t \\
& +O\left(T^{\varepsilon}\right) \\
= & -2 \sum_{d \leq N} \sum_{\substack{n_{1}, n_{2} \leq \frac{N}{d} \\
\left(n_{1}, n_{2}\right)=1}} \frac{a_{d n_{1}} \bar{a}_{d n_{2}}}{d n_{1} n_{2}} \int_{\mathbb{R}} \int_{0}^{\infty} \sum_{|\Delta| \neq 0} \frac{1}{\Delta^{2}} \mathrm{e}\left(\frac{\Delta y}{2 \pi}\right) \frac{d^{2}}{d y^{2}}\left(W\left(\frac{2 \pi t}{n_{1} n_{2} y^{2}}\right) \frac{1}{y}\right) d y \phi\left(\frac{t}{T}\right) d t \\
& +O\left(T^{\varepsilon}\right) \\
= & \mathcal{A}_{0,+}+\mathcal{A}_{0,-}+O\left(T^{\varepsilon}\right),
\end{aligned}
$$


where we can take the sum over $\Delta$ inside the integrals since they converge absolutely, and after a change of variables,

$$
\begin{aligned}
\mathcal{A}_{0, \pm} & =-2 \sum_{\substack { d \leq N \\
\begin{subarray}{c}{n_{1}, n_{2} \leq \frac{N}{d} \\
\left(n_{1}, n_{2}\right)=1{ d \leq N \\
\begin{subarray} { c } { n _ { 1 } , n _ { 2 } \leq \frac { N } { d } \\
( n _ { 1 } , n _ { 2 } ) = 1 } }\end{subarray}} \frac{a_{d n_{1}} \bar{a}_{d n_{2}}}{d n_{1} n_{2}} \int_{\mathbb{R}} \int_{0}^{\infty} \sum_{\Delta=1}^{\infty}\left(e^{i y}+e^{-i y}\right) \frac{d^{2}}{d y^{2}}\left(W\left(\frac{2 \pi \Delta^{2} t}{n_{1} n_{2} y^{2}}\right) \frac{1}{y}\right) d y \phi\left(\frac{t}{T}\right) d t \\
& =-2 \sum_{n_{1}, n_{2} \leq N} \frac{a_{n_{1}} \bar{a}_{n_{2}}\left(n_{1}, n_{2}\right)}{n_{1} n_{2}} \int_{\mathbb{R}} \int_{0}^{\infty} \sum_{\Delta=1}^{\infty} 2 \cos (y) \frac{d^{2}}{d y^{2}}\left(W\left(\frac{2 \pi \Delta^{2} t}{n_{1}^{*} n_{2}^{*} y^{2}}\right) \frac{1}{y}\right) d y \phi\left(\frac{t}{T}\right) d t,
\end{aligned}
$$

where we recall that $n_{i}^{*}=\frac{n_{i}}{\left(n_{1}, n_{2}\right)}$ for $i=1,2$. We notice that

$$
\begin{aligned}
\sum_{\Delta=1}^{\infty} \frac{d^{2}}{d y^{2}}\left(W\left(\frac{2 \pi \Delta^{2} t}{n_{1}^{*} n_{2}^{*} y^{2}}\right) \frac{1}{y}\right) & =\frac{1}{2 \pi i} \sum_{\Delta=1}^{\infty} \int_{(2)}\left(\frac{2 \pi \Delta^{2} t}{n_{1}^{*} n_{2}^{*}}\right)^{-w}(2 w-1)(2 w-2) y^{2 w-3} G(w) \frac{d w}{w} \\
& =\frac{1}{2 \pi i} \int_{\left(\frac{5}{4}\right)} \zeta(2 w)\left(\frac{2 \pi t}{n_{1}^{*} n_{2}^{*}}\right)^{-w}(2 w-1)(2 w-2) y^{2 w-3} G(w) \frac{d w}{w}
\end{aligned}
$$

For $0<\Re(s)<1$, we have

$$
\int_{0}^{\infty} \cos (y) y^{s-1} d y=\Gamma(s) \cos \left(\frac{\pi s}{2}\right)
$$

(see, for example, GR07, formula 3.381, 5., page 346), whence we are left with

$$
\frac{-2}{2 \pi i} \int_{\left(\frac{1}{4}\right)} \Gamma(2 w) \cos (\pi w) \zeta(2 w)\left(\frac{2 \pi t}{n_{1}^{*} n_{2}^{*}}\right)^{-w} G(w) \frac{d w}{w}
$$

where we used the multiplication formula for the gamma function, the identity $\cos (x-$ $\pi)=-\cos (x)$, and we moved the line of integration without encountering any pole, due to the assumption that $G(w)$ vanishes at $w=\frac{1}{2}$. Thus,

$$
\begin{aligned}
& \mathcal{A}_{0}+O\left(T^{\varepsilon}\right) \\
& =\frac{2}{2 \pi i} \sum_{n_{1}, n_{2}} \frac{a_{n_{1}} \bar{a}_{n_{2}}\left(n_{1}, n_{2}\right)}{n_{1} n_{2}} \int_{\mathbb{R}} \int_{\left(\frac{1}{4}\right)} 2 \cos (\pi w) \Gamma(2 w) \zeta(2 w)\left(\frac{2 \pi t}{n_{1}^{*} n_{2}^{*}}\right)^{-w} G(w) \frac{d w}{w} \phi\left(\frac{t}{T}\right) d t \\
& =\frac{-2}{2 \pi i} \sum_{n_{1}, n_{2}} \frac{a_{d n_{1}} \bar{a}_{n_{2}}\left(n_{1}, n_{2}\right)}{n_{1} n_{2}} \int_{\mathbb{R}} \int_{\left(-\frac{1}{4}\right)} \zeta(1+2 w)\left(\frac{t}{2 \pi n_{1}^{*} n_{2}^{*}}\right)^{w} G(w) \frac{d w}{w} \phi\left(\frac{t}{T}\right) d t
\end{aligned}
$$

where we used the functional equation of the Riemann zeta function (e.g. Chapter 10 in [Dav00]), and then we made the change of variables $w \rightarrow-w$ and use the fact that $G(w)=G(-w)$. 
From (3.1), we have

$$
\begin{aligned}
\mathcal{D}+\mathcal{A}_{0}= & \frac{2}{2 \pi i} \sum_{n_{1}, n_{2}} \frac{a_{n_{1}} \bar{a}_{n_{2}}\left(n_{1}, n_{2}\right)}{n_{1} n_{2}} \int_{\mathbb{R}} \int_{(2)}\left(\frac{t}{2 \pi n_{1}^{*} n_{2}^{*}}\right)^{w} \zeta(1+2 w) G(w) \frac{d w}{w} \phi\left(\frac{t}{T}\right) d t \\
& -\frac{2}{2 \pi i} \sum_{n_{1}, n_{2}} \frac{a_{n_{1}} \bar{a}_{n_{2}}\left(n_{1}, n_{2}\right)}{n_{1} n_{2}} \int_{\mathbb{R}} \int_{(-1 / 4)}\left(\frac{t}{2 \pi n_{1}^{*} n_{2}^{*}}\right)^{w} \zeta(1+2 w) G(w) \frac{d w}{w} \phi\left(\frac{t}{T}\right) d t+O\left(T^{\varepsilon}\right) \\
= & \sum_{n_{1}, n_{2}} \frac{a_{n_{1}} \bar{a}_{n_{2}}\left(n_{1}, n_{2}\right)}{n_{1} n_{2}} \int_{\mathbb{R}}\left(\log \frac{t}{2 \pi n_{1}^{*} n_{2}^{*}}+2 \gamma\right) \phi\left(\frac{t}{T}\right) d t+O\left(T^{\varepsilon}\right),
\end{aligned}
$$

since

$$
\operatorname{Res}_{w=0} x^{w} \zeta(1+2 w) \frac{G(w)}{w}=\frac{1}{2} \log x+\gamma .
$$

Now we have the main term. The rest of the off-diagonal terms contribute to the error term as shown in the following two cases.

Case 2: $|h| \geq \frac{N_{2}}{d M} T^{\varepsilon}$. In this case and in Case 3, we define $H_{d}:=\frac{N_{2}}{d M} T^{\varepsilon}$. By changing variable $t=x y$, we have

$$
\frac{1}{n_{1} n_{2}} \widetilde{\mathcal{A}}_{M, N_{i}}\left(h, n_{1}, n_{2}, \Delta\right)=\frac{1}{n_{1} n_{2}} \int_{\mathbb{R}} \mathrm{e}\left(\frac{\Delta y}{2 \pi}\right) \int_{0}^{\infty} \mathrm{e}\left(-\frac{h x}{n_{1} n_{2}}\right) F_{M}\left(\frac{x}{n_{1}}\right) W\left(\frac{2 \pi x}{n_{1} n_{2} y}\right) \phi\left(\frac{y x}{T}\right) d x d y .
$$

Since $F_{M}$ is supported in $[M / 2,3 M], x \asymp \frac{N_{1} M}{d}$. Moreover, $\frac{y}{T} \asymp \frac{1}{x} \asymp \frac{d}{N_{1} M}$ due to the support of $\phi$, and $\frac{1}{n_{1} n_{2} y} \ll \frac{T^{\varepsilon} 1}{x} \asymp \frac{d T^{\varepsilon_{1}}}{N_{1} M}$ because of the rapid decay of $W$. Hence integrating by parts $\ell+1$ times, for $T \leq t \leq 2 T$ we have

$$
\begin{aligned}
& \frac{1}{n_{1} n_{2}} \int_{0}^{\infty} \mathrm{e}\left(-\frac{h x}{n_{1} n_{2}}\right) F_{M}\left(\frac{x}{n_{1}}\right) W\left(\frac{2 \pi x}{n_{1} n_{2} y}\right) \phi\left(\frac{y x}{T}\right) d x \\
& \ll_{\ell, \varepsilon} \frac{d^{2}}{N_{1} N_{2}}\left(\frac{n_{1} n_{2}}{h} \frac{d T^{\varepsilon_{1}}}{M N_{1}}\right)^{\ell+1} \frac{M N_{1}}{d} \\
& \ll\left(\frac{T^{\varepsilon_{1}}}{h}\right)^{\ell+1}\left(\frac{N_{2}}{d M}\right)^{\ell} .
\end{aligned}
$$

Therefore, the contribution to $\mathcal{S}$ when $|h|>H_{d}$ is

$$
\ll \sum_{d \leq N} \sum_{\substack{N_{1}, N_{2} \leq N, M \leq T^{1 / 2+\varepsilon} \sqrt{\frac{N_{2}}{N_{1}}}}}^{\prime} \sum_{0<|\Delta| \leq \frac{D}{d}} \sum_{\substack{n_{1}, n_{2} \\\left(n_{1}, n_{2}\right)=1}} \frac{a_{d n_{1}} \bar{a}_{d n_{2}} F_{N_{1}}\left(d n_{1}\right) F_{N_{2}}\left(d n_{2}\right)}{d} \sum_{|h| \geq H_{d}} \frac{d T}{h N_{1} M}\left(\frac{N_{2} T^{\varepsilon_{1}}}{d M h}\right)^{\ell}
$$
$\ll T^{-A}$,

when $\ell$ is sufficiently large. Thus, the terms $|h|>H_{d}$ give a negligible contribution. 
Case 3: $0<|h|<H_{d}$. It is sufficient to consider the terms $0<h<H_{d}$. By changing variables $t=y x$, and $x$ to $x n_{1} n_{2}$, we will consider the dyadic contribution

$$
\begin{aligned}
\mathcal{A}_{M, N_{1}, N_{2}}^{*}:= & \sum_{\substack{n_{1}, n_{2} \\
\left(n_{1}, n_{2}\right)=1}} \sum_{0<|\Delta| \leq \frac{D}{d}} \sum_{0<h<H_{d}} \frac{a_{d n_{1}} \bar{a}_{d n_{2}} F_{N_{1}}\left(d n_{1}\right) F_{N_{2}}\left(d n_{2}\right)}{d} \times \\
& \times \mathrm{e}\left(-\frac{h \Delta \bar{n}_{1}}{n_{2}}\right) \int_{0}^{\infty} \mathrm{e}(-h x) F_{M}\left(x n_{2}\right) \int_{\mathbb{R}} \mathrm{e}\left(\frac{\Delta y}{2 \pi}\right) W\left(\frac{2 \pi x}{y}\right) \phi\left(\frac{y x n_{1} n_{2}}{T}\right) d y d x .
\end{aligned}
$$

We write $\phi$ in term of its Mellin transform $\widetilde{\phi}$, to separate the variables $n_{1}$ and $n_{2}$. Let $h \Delta=a, A=\frac{D H_{d}}{d}=\frac{N_{1} N_{2}}{d^{2} T^{1-\varepsilon}}$, and $\nu_{x, y}(a)=\sum_{h \Delta=a} \mathrm{e}\left(-h x+\frac{\Delta y}{2 \pi}\right)$. Therefore we have

$$
\begin{aligned}
\mathcal{A}_{M, N_{1}, N_{2}}^{*}= & \frac{1}{(2 \pi i) d} \int_{0}^{\infty} \int_{\mathbb{R}} \int_{(\varepsilon)} W\left(\frac{2 \pi x}{y}\right) \sum_{0<|a|<A} \nu_{x, y}(a) \times \\
& \times \sum_{\substack{n_{1}, n_{2} \\
\left(n_{1}, n_{2}\right)=1}} \frac{a_{d n_{1}} \bar{a}_{d n_{2}} F_{N_{1}}\left(d n_{1}\right) F_{N_{2}}\left(d n_{2}\right) F_{M}\left(x n_{2}\right)}{n_{1}^{w} n_{2}^{w}} \mathrm{e}\left(-\frac{a \bar{n}_{1}}{n_{2}}\right) \widetilde{\phi}(w) \frac{T^{w}}{x^{w} y^{w}} d w d y d x .
\end{aligned}
$$

Since $F_{M}$ is supported in $[M / 2,3 M], x \asymp \frac{d M}{N_{2}}$. Moreover, $y \asymp \frac{T}{x n_{1} n_{2}} \asymp \frac{T d}{M N_{1}}$ because $\phi$ is supported in $[1,2]$. Thus, using Proposition [1, we have

$$
\begin{aligned}
\mathcal{A}_{M, N_{1}, N_{2}}^{*} & \ll \frac{1}{d} \int_{x \asymp \frac{d M}{N_{2}}} \int_{y \asymp \frac{T d}{M N_{1}}}\left(\frac{\left(N_{1} N_{2} A\right)^{\frac{17}{20}+\varepsilon}}{d^{\frac{17}{10}-\varepsilon}} \frac{\left(N_{1}+N_{2}\right)^{\frac{1}{4}}}{d^{\frac{1}{4}}}+\frac{\left(N_{1} N_{2} A\right)^{\frac{7}{8}+\varepsilon}}{d^{\frac{7}{4}-\varepsilon}} \frac{\left(A N_{1}+A N_{2}\right)^{\frac{1}{8}}}{d^{\frac{1}{8}}}\right) d y d x \\
& \ll\left(T^{\frac{3}{20}+\varepsilon} \frac{\left(N_{1} N_{2}\right)^{\frac{7}{10}}}{d^{\frac{53}{20}}}\left(N_{1}+N_{2}\right)^{\frac{1}{4}}+T^{\varepsilon} \frac{\left(N_{1} N_{2}\right)^{\frac{7}{8}}}{d^{\frac{23}{8}}}\left(N_{1}+N_{2}\right)^{\frac{1}{8}}\right)
\end{aligned}
$$

Summing over dyadic intervals for $M \leq T^{1 / 2+\varepsilon} \sqrt{\frac{N_{2}}{N_{1}}}, N_{i} \leq N$, and $d \leq N$, we have that the contribution to $\mathcal{A}$ when $|h| \leq H_{d}$ is bounded by $T^{\frac{3}{20}+\varepsilon} N^{\frac{33}{20}}+T^{\varepsilon} N^{\frac{15}{8}}$. Therefore we take $N$ up to $T^{\frac{17}{33}-\varepsilon}$ to obtain an eligible error term in Theorem 1,

3.3. A trivial bound for $\mathcal{E}$. Extracting the common divisor $d$ from $n_{1}$ and $n_{2}$, applying Poisson summation formula, and changing variables, we can write (3.5) as

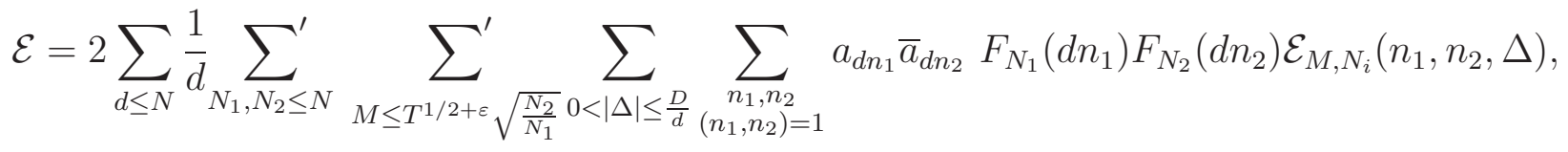

where

$$
\begin{aligned}
\mathcal{E}_{M, N_{i}}\left(n_{1}, n_{2}, \Delta\right)= & \frac{1}{n_{1} n_{2}} \sum_{h \in \mathbb{Z}} \mathrm{e}\left(-\frac{h \bar{n}_{1} \Delta}{n_{2}}\right) \int_{0}^{\infty} \mathrm{e}\left(-\frac{h x}{n_{1} n_{2}}\right) F_{M}\left(\frac{x}{n_{1}}\right) \int_{\mathbb{R}} \mathrm{e}\left(\frac{\Delta t}{2 \pi x}\right) \phi\left(\frac{t}{T}\right) \times \\
& \times\left[W\left(\frac{2 \pi x^{2}}{n_{1} n_{2} t}\right)\left(-\frac{\Delta}{2 x^{2}}-\frac{i t \Delta^{2}}{2 x^{3}}\right)+\frac{2 \pi \Delta}{n_{1} n_{2} t} W^{\prime}\left(\frac{2 \pi x^{2}}{n_{1} n_{2} t}\right)\right] d t d x
\end{aligned}
$$


Integrating by parts, as in Case 2 of the previous section, we see that the contribution coming from the terms with $|h|>H_{d}$ is $O(1)$. Thus, estimating trivially the rest of the terms we have

$$
\mathcal{E}_{M, N_{i}}\left(n_{1}, n_{2}, \Delta\right) \ll \frac{T^{\varepsilon}}{n_{1} n_{2}}\left(1+\frac{N_{2}}{d M}\right),
$$

whence

$$
\mathcal{E} \ll T^{-1 / 2+\varepsilon} N+T^{-1+\varepsilon} N^{2} \ll N T^{\varepsilon}
$$

and the proof of Theorem 1 is complete.

3.4. The proof of Theorem 2. The proof of Theorem 2 is the same as Theorem 1 except that we use (1.3) instead of Proposition 1 in (3.7). Notice that (1.3) is applicable, since $A=\frac{N_{1} N_{2}}{d^{2} T^{1-\varepsilon}} \leq\left(\frac{N_{1} N_{2}}{d^{2}}\right)^{\frac{0.5-r}{1+2 t}+\varepsilon}$ by $\frac{N_{i}}{d} \leq N \leq T^{\frac{1}{2}+\frac{0.5-r}{1+2(r+2 t)}}$. Thus, we obtain that

$$
\begin{aligned}
\mathcal{A}_{M, N_{1}, N_{2}}^{*} & \ll \frac{T^{\varepsilon}}{d} \int_{x \asymp \frac{d M}{N_{2}}} \int_{y \asymp \frac{T d}{M N_{1}}} \frac{\sqrt{N_{1} N_{2} A}}{d}\left(\frac{\left(N_{1}+N_{2}\right)^{\frac{1}{2}+r} A^{t}}{d^{\frac{1}{2}+r}}\right)+\frac{A N_{1}}{d}+\frac{A N_{2}}{d} d y d x \\
& \ll T^{\frac{1}{2}+\varepsilon-t}\left(\frac{\left(N_{1}+N_{2}\right)^{\frac{1}{2}+r}\left(N_{1} N_{2}\right)^{t}}{d^{\frac{3}{2}+r+2 t}}\right)+\frac{N_{1}}{d^{2}}+\frac{N_{2}}{d^{2}} .
\end{aligned}
$$

Summing over dyadic intervals for $M, N_{i}$, we have that the contribution to $\mathcal{A}$ from these terms is $T^{\frac{1}{2}+\varepsilon-t} N^{\frac{1}{2}+r+2 t}+N^{1+\varepsilon}$ and Theorem 2 follows.

\section{Proof of Theorem 3}

The proof of Theorem 3 follows the proof of Theorem 1 except the last part when $0<|h|<H_{d}$. Here we only modify the last part of the proof using the same arguments by Deshouillers and Iwaniec in [DI84]. By the same change of variables, we have to consider

$$
\begin{aligned}
\mathcal{A}_{M, N_{1}, N_{2}}^{*} & =\sum_{\substack{n_{1}, n_{2} \\
\left(n_{1}, n_{2}\right)=1}} \sum_{0<|\Delta| \leq \frac{D}{d}} \sum_{0<|h|<H_{d}} a_{d n_{1}} \bar{a}_{d n_{2}} F_{N_{1}}\left(d n_{1}\right) F_{N_{2}}\left(d n_{2}\right) \times \\
& \times \mathrm{e}\left(-\frac{h \bar{n}_{1} \Delta}{n_{2}}\right) \int_{0}^{\infty} \mathrm{e}(-h x) F_{M}\left(x n_{2}\right) \int_{\mathbb{R}} \mathrm{e}\left(\frac{\Delta y}{2 \pi}\right) W\left(\frac{2 \pi x}{y}\right) \phi\left(\frac{x y n_{1} n_{2}}{T}\right) d y d x .
\end{aligned}
$$

We now write $a_{d n_{1}}$ as $\alpha_{\mu j} \beta_{\nu r}$, where $\mu \mid d^{\infty},(d, j)=1, n_{1}=\varrho r j, \nu=\frac{d}{(\mu, d)}$ and $\varrho=\frac{\mu}{(\mu, d)}$. Therefore, we have to bound

$$
\sum_{d \leq T} \frac{1}{d} \sum_{N_{1}, N_{2}, M}^{\prime} \sum_{\substack{\mu \mid d^{\infty} \\ \nu=d /(\mu, d)}} \sum_{0<|\Delta| \leq \frac{D}{d}} \sum_{0<|h|<H_{d}} \sum_{\left(n_{2}, \varrho\right)=1} \bar{a}_{d n_{2}} \sum_{\left(j, d n_{2}\right)=1} \alpha_{\mu j} \sum_{\left(r, n_{2}\right)=1} \beta_{\nu r} \mathrm{e}\left(-\frac{h \Delta \overline{\varrho r j}}{n_{2}}\right)
$$

$$
\times F_{N_{1}}(d \varrho r j) F_{N_{2}}\left(d n_{2}\right) \int_{0}^{\infty} \mathrm{e}(-h x) F_{M}\left(x n_{2}\right) \int_{\mathbb{R}} \mathrm{e}\left(\frac{\Delta y}{2 \pi}\right) W\left(\frac{2 \pi x}{y}\right) \phi\left(\frac{x y \varrho r j n_{2}}{T}\right) d y d x,
$$

where the sums over $N_{1}, N_{2}, M$ are dyadic sums up to $N K, N K$, and $T^{1 / 2+\epsilon} \sqrt{N_{2} / N_{1}}$. 
To bound the above sum, we use Proposition 2. However, first we need to apply Mellin's transform to $F_{N_{1}}$ and $\phi$ to separate variables $n_{2}, r, j$. The technique is standard, so we skip the details. From Proposition 2, the sum over $\ell$ is the sum over $n_{2}$, and $L=\frac{N_{2}}{d}$. The sum over $j$ is the sum over $j$, and $J \leq \frac{N}{\mu}$. The sum over $u$ is the sum over $h \Delta$, and $U=\frac{N_{1} N_{2}}{d^{2} T^{1-2 \varepsilon}}$. Finally the sum over $v$ is the sum over $r$, and $V \leq \frac{K}{\nu}$. Moreover, we note that $J V \leq \frac{N_{1}}{d \varrho}$. Applying Proposition 2, we obtain that (4.1) is bounded by (after summing over dyadic $M$ )

$$
\begin{aligned}
& \ll T^{\varepsilon} \sum_{d \leq T} \sum_{\substack{\mu \mid d^{\infty} \\
\nu=d /(\mu, d)}} \sum_{N_{1}}^{\prime} \sum_{N_{2}}^{\prime} \frac{d T}{N_{1} N_{2}}\left(\frac{N_{1} N_{2}}{\varrho^{1 / 2} d^{2} T^{1 / 2}}\right)\left\{\left(\frac{N_{2} N}{d \mu}\right)^{1 / 2}+\left(\frac{N_{1} N_{2}}{d^{2} T}+\frac{K}{\nu}\right)^{1 / 4} \times\right. \\
& \left.\times\left[\frac{N_{2} N}{d \mu}\left(\frac{N_{1} N_{2}}{T d^{2}}+\frac{\varrho K}{\nu}\right)\left(\frac{N_{2}}{d}+\frac{\varrho K^{2}}{\nu^{2}}\right)+\frac{\varrho N_{1} N_{2} N^{2} K^{2}}{d^{2} T \mu^{2} \nu^{2}}\right]^{1 / 4}\right\} \\
& \ll \sum_{d \leq T} \sum_{\substack{\mu \mid d^{\infty} \\
\nu=d /(\mu, d)}} \frac{T^{\frac{1}{2}+\varepsilon}}{\varrho^{1 / 2} d}\left\{\left(\frac{N^{2} K}{d \mu}\right)^{1 / 2}+\left(\frac{N^{2} K^{2}}{d^{2} T}+\frac{K}{\nu}\right)^{1 / 4} \times\right. \\
& \left.\times\left[\frac{N^{2} K}{d \mu}\left(\frac{N^{2} K^{2}}{T d^{2}}+\frac{\varrho K}{\nu}\right)\left(\frac{N K}{d}+\frac{\varrho K^{2}}{\nu^{2}}\right)+\frac{\varrho N^{4} K^{4}}{d^{2} T \mu^{2} \nu^{2}}\right]^{1 / 4}\right\} \\
& \ll T^{\varepsilon}\left(T^{1 / 2} N^{3 / 4} K+T^{1 / 2} N K^{1 / 2}+N^{7 / 4} K^{3 / 2}\right) \sum_{d \leq T} \sum_{\substack{\mu \mid d^{\infty} \\
\nu=d /(\mu, d)}} \frac{1}{d^{5 / 4}} \frac{1}{\mu^{1 / 4}} \\
& \ll T^{\varepsilon}\left(T^{1 / 2} N^{3 / 4} K+T^{1 / 2} N K^{1 / 2}+N^{7 / 4} K^{3 / 2}\right),
\end{aligned}
$$

and this completes the proof of Theorem 3 .

\section{Proof of Theorem 4}

The proof of Theorem 4 follows the proof of Theorem 1 except the last part when $0<|h|<H_{d}$.

We recall that we have $a_{b}=\sum_{n k=b} \alpha_{n} \beta_{k}$, and we assume that $\alpha_{n}$ is supported on $\left[N T^{-\xi_{1}}, 2 N\right]$, where $N \ll T^{\frac{1}{2}+\varepsilon}$ and $0 \leq \xi_{1} \leq \frac{1}{5}$. Moreover $\beta_{k}$ is supported on $k \in$ $\left[K T^{-\xi_{2}}, 2 K\right]$, and $0 \leq \xi_{1} \leq \frac{1}{16}$. Let $\xi_{1}+\xi_{2}=\xi$. We introduce smooth partitions of unity in the sums over $n$ and $k$ (without indicating it, to save notation). Thus, $n_{i} \approx N_{i}$, $k_{i} \approx K_{i}$ (note that in the notation of Section $3 N_{i}$ was the size of $b=n k$, so $N_{i} K_{i}$ in the current notation). Thus, $d b_{i} \asymp N_{i} K_{i}$. Moreover, we assume that $\alpha_{n}=\psi(n)$, where $\psi(x)$ is a smooth function such that $\psi^{(j)}(x) \ll_{j} x^{-j}$ for $j \geq 0$. 
We have to bound

$$
\begin{aligned}
\mathcal{A}_{N, K}^{\prime}:= & \sum_{d \leq N K} \sum_{N_{1}, N_{2}, K_{1}, K_{2}}^{\prime} \sum_{M \leq T^{\frac{1}{2}+\varepsilon}}^{\prime} \sum_{\sqrt{\frac{N_{2} K_{2}}{N_{1} K_{1}}}} \sum_{\substack{b_{1}, b_{2} \\
\left(b_{1}, b_{2}\right)=1}} \sum_{0<|\Delta| \leq \frac{M N_{1} K_{1}}{d T^{1-\varepsilon}}} \sum_{0<|h|<\frac{N_{2} K_{2} T^{\varepsilon}}{d M}} \mathrm{e}\left(-\frac{h \bar{b}_{1} \Delta}{b_{2}}\right) \int_{0}^{\infty} \mathrm{e}\left(-\frac{h x}{b_{1} b_{2}}\right) \frac{F_{M}\left(\frac{x}{b_{1}}\right)}{x} \times \\
& \frac{a_{d b_{1}} \overline{a_{d b_{2}}} F_{N_{1} K_{1}}\left(d b_{1}\right) F_{N_{2} K_{2}}\left(d b_{2}\right)}{d b_{1} b_{2}} \mathrm{e} \\
& \times \int_{\mathbb{R}} \mathrm{e}\left(\frac{\Delta t}{2 \pi x}\right) W\left(\frac{2 \pi x^{2}}{b_{1} b_{2} t}\right) \phi\left(\frac{t}{T}\right) d t d x .
\end{aligned}
$$

After the change of variables $y=\frac{t}{2 \pi x}$ and then $z=\frac{x}{b_{1} b_{2}}$, it becomes

$$
\begin{aligned}
\mathcal{A}_{N, K}^{\prime}= & 2 \pi \sum_{d \leq N K} \sum_{N_{1}, N_{2}, K_{1}, K_{2}}^{\prime} \sum_{M \leq T^{\frac{1}{2}+\varepsilon}}^{\prime} \sum_{\sqrt{\frac{N_{2} K_{2}}{N_{1} K_{1}}}} \sum_{b_{1}, b_{2}} \sum_{0<|\Delta| \leq \frac{M N_{1} K_{1}}{d T^{1-\varepsilon}}} \sum_{0<|h|<\frac{N_{2} K_{2} T^{\varepsilon}}{d M}} \\
& \frac{a_{d b_{1}} \overline{a_{d b_{2}}} F_{N_{1} K_{1}}\left(d b_{1}\right) F_{N_{2} K_{2}}\left(d b_{2}\right)}{d} \mathrm{e}\left(-\frac{h \bar{b}_{1} \Delta}{b_{2}}\right) \int_{0}^{\infty} \mathrm{e}(-h z) F_{M}\left(z b_{2}\right) \times \\
& \times \int_{\mathbb{R}} \mathrm{e}(\Delta y) W\left(\frac{z}{y}\right) \phi\left(\frac{2 \pi y z b_{1} b_{2}}{T}\right) d y d z,
\end{aligned}
$$

Firstly we claim that we can truncate the sum over $d$ at height $Y:=\left(N_{1} K_{1} N_{2} K_{2}\right)^{\frac{1}{2}} / T^{\frac{1}{2}+\eta}$ for some small $\eta>0$, up to an error term with a power saving. This is because for larger values of $d$ we are essentially left with the contribution coming from a Dirichlet polynomial of length $T^{\frac{1}{2}+\eta}$, which we can bound using the method used to prove Theorem 1. More precisely, by (3.7), we have that the contribution from the terms with $d \geq Y$ is bounded by

$$
\begin{aligned}
& \ll \sum_{N_{1}, N_{2}, K_{1}, K_{2}}^{\prime} \sum_{Y \leq d \leq N K}\left(\frac{T^{\frac{3}{20}+\varepsilon}\left(N_{1} K_{1}+N_{2} K_{2}\right)^{\frac{1}{4}}\left(N_{1} K_{1} N_{2} K_{2}\right)^{\frac{7}{10}}}{d^{\frac{53}{20}}}+\right. \\
& \left.+T^{\varepsilon} \frac{\left(N_{1} K_{1}+N_{2} K_{2}\right)^{\frac{1}{8}}\left(N_{1} K_{1} N_{2} K_{2}\right)^{\frac{7}{8}}}{d^{\frac{23}{8}}}\right) \\
& \ll \sum_{N_{1}, N_{2}, K_{1}, K_{2}}^{\prime}\left(T^{\frac{39}{40}+\frac{33}{20} \eta+\varepsilon}\left(\frac{N_{1} K_{1}}{N_{2} K_{2}}+\frac{N_{2} K_{2}}{N_{1} K_{1}}\right)^{\frac{1}{8}}+T^{\frac{15}{16}+\frac{15}{8} \eta+\varepsilon}\left(\frac{N_{1} K_{1}}{N_{2} K_{2}}+\frac{N_{2} K_{2}}{N_{1} K_{1}}\right)^{\frac{1}{16}}\right) \\
& \ll T^{\frac{39}{40}+\frac{33}{20} \eta+\frac{1}{8} \xi+\varepsilon}+T^{\frac{15}{16}+\frac{15}{8} \eta+\frac{1}{16} \xi+\varepsilon},
\end{aligned}
$$

since $T^{-\xi} \ll \frac{N_{1} K_{1}}{N_{2} K_{2}} \ll T^{\xi}$.

For the remaining part of the proof we use Watt's arguments in Wat95. We write

$$
a_{d b_{i}}=\sum_{h_{i} k_{i}=d b_{i}} \alpha_{h_{i}} \beta_{k_{i}}=\sum_{\substack{f_{i} g_{i}=b_{i}, \mu_{i} \nu_{i}=d,\left(g_{i}, \frac{d}{\nu_{i}}\right)=1}} \alpha_{f_{i} \mu_{i}} \beta_{g_{i} \nu_{i}},
$$


so that $f_{i} \asymp N_{i} / \mu_{i}$ and $g_{i} \asymp K_{i} / \nu_{i}$. We will apply Proposition 3 to bound

$$
\begin{aligned}
& \sum_{M \leq T^{\frac{1}{2}+\varepsilon} \sqrt{\frac{N_{2} K_{2}}{N_{1} K_{1}}}}^{\prime} \sum_{d \leq Y} \frac{1}{d} \sum_{\substack{\mu_{1}, \nu_{1}, \mu_{2}, \nu_{2}, d \\
\mu_{1} \nu_{1}=\mu_{2} \nu_{2}=d}} \int_{z \asymp \frac{d M}{N_{2} K_{2}}} \int_{y \asymp \frac{d T}{M N_{1} K_{1}}} \mid W\left(\frac{z}{y}\right) \times \\
& \times \sum_{\substack{g_{1}, g_{2} \\
\left(g_{i}, \frac{d}{\nu_{i}}\right)=1}} \sum_{\substack{f_{1}, f_{2} \\
\left(f_{1} g_{1}, f_{2} g_{2}\right)=1}} \sum_{0<|\Delta| \leq \frac{M N_{1} K_{1}}{d T^{1-\varepsilon}}} \sum_{0<|h|<\frac{N_{2} K_{2} T^{\varepsilon}}{d M}} \alpha_{f_{1} \mu_{1}} \beta_{g_{1} \nu_{1}} \overline{\alpha_{f_{2} \mu_{2}} \beta_{g_{2} \nu_{2}}} \mathrm{e}\left(\frac{h \Delta \overline{f_{1} g_{1}}}{f_{2} g_{2}}\right) \\
& \times F_{N_{1} K_{1}}\left(f_{1} g_{1} \mu_{1} \nu_{1}\right) F_{N_{2} K_{2}}\left(f_{2} g_{2} \mu_{2} \nu_{2}\right) \mathrm{e}(-h z) \mathrm{e}(\Delta y) F_{M}\left(z f_{2} g_{2}\right) \phi\left(\frac{2 \pi y z f_{1} f_{2} g_{1} g_{2}}{T}\right) \mid d y d z
\end{aligned}
$$

Before using Proposition 3 (with $H=\frac{M N_{1} K_{1}}{d T^{1-\varepsilon}}, C=\frac{N_{2} K_{2} T^{\varepsilon}}{d M}, r=g_{1}, s=g_{2}, v=f_{1}, p=$ $f_{2}$ in the proposition respectively), we verify that $X^{2}=\frac{N_{1} N_{2} K_{1} K_{2}}{H C d^{2}}=T^{1-\varepsilon} \gg T^{\varepsilon}$, which is clearly satisfied if $\varepsilon$ is small enough, and that

$$
\left(\frac{K_{1} K_{2}}{\nu_{1} \nu_{2}}\right)^{2} \geq \frac{N_{1}^{2} K_{1}^{2} N_{2} K_{2} M}{d^{3} T^{2-\varepsilon}}, \quad\left(\frac{K_{1} K_{2}}{\nu_{1} \nu_{2}}\right)^{2} \geq \frac{N_{2} K_{2} \mu_{1}}{N_{1} \mu_{2} \nu_{2}} T^{\varepsilon}=\frac{K_{2} N_{2} \mu_{1}}{d N_{1}} T^{\varepsilon}=\frac{K_{2} N_{2}}{\nu_{1} N_{1}} T^{\varepsilon} .
$$

Since $M \leq T^{\frac{1}{2}+\varepsilon}\left(N_{2} K_{2} / N_{1} K_{1}\right)^{\frac{1}{2}}$, and $d \leq \frac{\left(N_{1} K_{1} N_{2} K_{2}\right)^{\frac{1}{2}}}{T^{\frac{1}{2}+\eta}}$, the first condition is implied by $T^{2-\varepsilon} \geq N_{1}^{2} N_{2}^{2} T^{\varepsilon-\eta}$, which is true if $2 \varepsilon<\eta$. The second condition is equivalent to $K_{1}^{2} K_{2} \geq \nu_{1} \nu_{2}^{2} T^{\varepsilon} \frac{N_{2}}{N_{1}}$. This is true as long as $\eta>\frac{1}{6} \xi_{2}+\frac{4}{3} \varepsilon$, since $N_{i} \leq T^{1 / 2+\varepsilon}$, $K_{2} / K_{1} \ll T^{\xi_{2}}$, and

$$
\nu_{1} \nu_{2}^{2} \frac{N_{2}}{N_{1}} T^{\varepsilon} \leq d^{3} \frac{N_{2}}{N_{1}} T^{\varepsilon} \leq \frac{N_{1}^{\frac{1}{2}} N_{2}^{\frac{5}{2}} K_{1}^{\frac{3}{2}} K_{2}^{\frac{3}{2}} T^{\varepsilon-3 \eta}}{T^{3 / 2}} \leq K_{1}^{2} K_{2} \frac{K_{2}^{\frac{1}{2}}}{K_{1}^{\frac{1}{2}}} T^{4 \varepsilon-3 \eta} \leq K_{1}^{2} K_{2} T^{4 \varepsilon-3 \eta+\frac{\xi_{2}}{2}} .
$$

Applying Proposition 3 with $\delta^{-1}=\max (z C, y H)+1 \ll T^{\varepsilon}$ and using that $\mu_{i}, \nu_{i} \leq$ $d \leq \frac{\left(N_{1} K_{1} N_{2} K_{2}\right)^{\frac{1}{2}}}{T^{\frac{1}{2}+\eta}}$, we obtain that $(\underline{5.2})$ is bounded by

$$
\begin{aligned}
& \sum_{M \leq T^{\frac{1}{2}+\varepsilon}}^{\prime} \sum_{\sqrt{\frac{N_{2} K_{2}}{N_{1} K_{1}}}} \frac{T^{\varepsilon}}{d} \sum_{\substack{\mu_{1}, \nu_{1}, \mu_{2}, \nu_{2}, \mu_{1} \nu_{1}=\mu_{2} \nu_{2}=d}} \frac{d M}{N_{2} K_{2}} \frac{d T}{M N_{1} K_{1}} \frac{N_{1} N_{2} K_{1} K_{2}}{T d^{2}} \frac{K_{1}}{\nu_{1}}\left(\frac{N_{1}}{\mu_{1}}+\frac{K_{2}}{\nu_{2}} T^{\frac{1}{2}}\right) \times \\
& \times\left(1+\frac{N_{1} N_{2} \nu_{1} \nu_{2}}{T d^{2}}\right)^{\frac{1}{2}}\left(1+\frac{d N_{2}}{\mu_{2} K_{1} N_{1}}\right)^{\frac{1}{2}}\left(1+\frac{M N_{1} N_{2}^{2} \nu_{1}^{3} \nu_{2}^{2}}{\left(K_{1} K_{2}\right)^{2} d T \mu_{2}^{2}}\right)^{\frac{1}{4}} \\
\ll & \sum_{M \leq T^{\frac{1}{2}+\varepsilon} \sqrt{\frac{N_{2} K_{2}}{N_{1} K_{1}}} d \leq Y}^{\prime} \frac{T^{\varepsilon}}{d} \sum_{\substack{\mu_{1}, \nu_{1}, \mu_{2}, \nu_{2}, \mu_{1} \nu_{1}=\mu_{2} \nu_{2}=d}} \frac{K_{1}}{\nu_{1}}\left(\frac{N_{1}}{\mu_{1}}+\frac{K_{2}}{\nu_{2}} T^{\frac{1}{2}}\right)\left(1+\frac{d N_{2}}{\mu_{2} K_{1} N_{1}}\right)^{\frac{1}{2}}\left(1+\frac{M N_{1} N_{2}^{2} \nu_{1}^{3} \nu_{2}^{2}}{\left(K_{1} K_{2}\right)^{2} d T \mu_{2}^{2}}\right)^{\frac{1}{4}} \\
\ll & \sum_{d \leq Y} \frac{T^{\varepsilon}}{d} \sum_{\substack{\mu_{1}, \nu_{1}, \mu_{2}, \nu_{2}, \mu_{1} \nu_{1}=\mu_{2} \nu_{2}=d}}\left(\frac{K_{1} K_{2} T^{\frac{1}{2}}}{\nu_{1} \nu_{2}}+\frac{K_{1} N_{1}}{d}+\frac{K_{1}^{\frac{3}{8}} N_{1}^{\frac{9}{8}} N_{2}^{\frac{5}{8}}}{\mu_{1}^{\frac{3}{4}} \mu_{2} K_{2}^{\frac{3}{8}} T^{\frac{1}{8}}}+\frac{K_{1}^{\frac{3}{8}} K_{2}^{\frac{5}{8}} T^{\frac{3}{8}} N_{1}^{\frac{1}{8}} N_{2}^{\frac{5}{8}}}{\nu_{1}^{\frac{1}{4}} d^{\frac{3}{4}}}+\right.
\end{aligned}
$$




$$
\begin{gathered}
\left.+\frac{K_{1}^{\frac{1}{2}} K_{2} T^{\frac{1}{2}} N_{2}^{\frac{1}{2}}}{\nu_{1} \nu_{2}^{\frac{1}{2}} N_{1}^{\frac{1}{2}}}+\frac{K_{1}^{\frac{1}{2}} N_{1}^{\frac{1}{2}} N_{2}^{\frac{1}{2}}}{d^{\frac{1}{2}} \mu_{2}^{\frac{1}{2}}}+\frac{N_{1}^{\frac{5}{8}} N_{2}^{\frac{9}{8}} d^{\frac{1}{2}}}{\mu_{1}^{\frac{3}{4}} \mu_{2}^{\frac{3}{2}} K_{2}^{\frac{3}{8}} K_{1}^{\frac{1}{8}} T^{\frac{1}{8}}}+\frac{T^{\frac{3}{8}} N_{2}^{\frac{9}{8}} K_{2}^{\frac{5}{8}}}{N_{1}^{\frac{3}{8}} K_{1}^{\frac{1}{8}} d^{\frac{1}{4}} \nu_{1}^{\frac{1}{4}} \mu_{2}^{\frac{1}{2}}}\right) \\
\ll K_{1} K_{2} T^{\frac{1}{2}+\varepsilon}+K_{1} N_{1} T^{\varepsilon}+\frac{K_{1}^{\frac{3}{8}} N_{1}^{\frac{9}{8}} N_{2}^{\frac{5}{8}}}{K_{2}^{\frac{3}{8}} T^{\frac{1}{8}-\varepsilon}}+K_{1}^{\frac{3}{8}} K_{2}^{\frac{5}{8}} T^{\frac{3}{8}+\varepsilon} N_{1}^{\frac{1}{8}} N_{2}^{\frac{5}{8}}+\frac{K_{1}^{\frac{1}{2}} K_{2} T^{\frac{1}{2}+\varepsilon} N_{2}^{\frac{1}{2}}}{N_{1}^{\frac{1}{2}}}+ \\
+K_{1}^{\frac{1}{2}} N_{1}^{\frac{1}{2}} N_{2}^{\frac{1}{2}} T^{\varepsilon}+\frac{N_{1}^{\frac{7}{8}} N_{2}^{\frac{11}{8}} K_{1}^{\frac{1}{8}}}{K_{2}^{\frac{1}{8}} T^{\frac{3}{8}+\frac{\eta}{2}-\varepsilon}}+\frac{T^{\frac{3}{8}}+\varepsilon}{N_{2}^{\frac{9}{8}} K_{2}^{\frac{5}{8}}} \\
N_{1}^{\frac{3}{8}} K_{1}^{\frac{1}{8}}
\end{gathered}
$$

Summing over $K_{i}$ and $N_{i}$, we obtain that (5.2) is bounded by

$$
K^{2} T^{\frac{1}{2}+\varepsilon}+T^{\frac{3}{4}+\frac{3 \xi_{2}}{8}+\varepsilon}+K N^{\frac{3}{4}} T^{\frac{3}{8}+\varepsilon}+K^{\frac{3}{2}} T^{\frac{1}{2}+\varepsilon+\frac{\xi_{1}}{2}}+T^{\frac{3}{4}+\frac{\xi_{2}}{8}-\frac{\eta}{2}+\varepsilon}+K^{\frac{1}{2}} N^{\frac{3}{4}} T^{\frac{3}{8}+\frac{3 \xi_{1}}{8}+\frac{\xi_{2}}{8}} .
$$

Theorem 4 then follows by taking $\eta=\frac{\xi_{2}}{6}+3 \varepsilon$ and collecting the error term (5.1).

\section{Proof of Corollary 2}

The proof of Corollary 2 requires the following two lemmas.

Lemma 2. Let $G$ be a compactly supported function. If $F=-G^{\prime}$ for $x>0$ and $F$ is three times continuously differentiable and compactly supported, then,

$$
\sum_{n} \frac{G\left(\frac{\log n}{\log x}\right)}{n^{s}}=\frac{1}{2 \pi i} \int_{(c)} \zeta(s+w) \cdot \widehat{F}\left(-\frac{i w \log x}{2 \pi}\right) \frac{d w}{w},
$$

for $c>\max (1-\Re(s), 0)$ and $x>1$, where $\widehat{F}$ denotes the Fourier transform of $F$,

$$
\widehat{F}(x):=\int_{-\infty}^{\infty} F(u) e^{-2 \pi i u x} d u
$$

Proof. First of all $\widehat{F}(x)$ is entire because $F$ is compactly supported. We expand the function $\zeta(s+w)$ into its Dirichlet series and compute

$$
\frac{1}{2 \pi i} \int_{(c)} n^{-w} \cdot \widehat{F}\left(-\frac{i w \log x}{2 \pi}\right) \frac{d w}{w} .
$$

Notice that

$$
\widehat{F}\left(-\frac{i w \log x}{2 \pi}\right)=\int_{-\infty}^{\infty} F(u) x^{u w} d u .
$$

Inserting this representation into (6.1) and inter-changing integrals, we obtain

$$
\int_{-\infty}^{\infty} F(u) \cdot \frac{1}{2 \pi i} \int_{(c)}\left(\frac{x^{u}}{n}\right)^{w} \cdot \frac{d w}{w} d u=\int_{\frac{\log n}{\log x}}^{\infty} F(u) d u=G\left(\frac{\log n}{\log x}\right) .
$$

In order to justify the interchange of the two integrations we truncate the integral in (6.1) at a large height $X$, committing an error which goes to zero as $X \rightarrow \infty$ (since the Fourier transform $\widehat{F}$ will decay sufficiently fast), and interchange. Then, we use a Perron formula with error term in order to compute the conditionally convergent 
Perron integral appearing above. Taking the height $X \rightarrow \infty$ returns the desired result, as stated.

Lemma 3. Let $A \geq 0$ and let $0<\eta<\frac{1}{66}$ be fixed constants. Let $v \in \mathbb{R}$ and $x<T^{1 / 2+\eta}$. Let $s=\sigma+i t$, where $\sigma=\frac{1}{2}+\frac{A}{\log T}$ and $T \leq t \leq 2 T$. Then

$$
\int_{T}^{2 T}|\zeta(s)|^{2} \cdot\left|\sum_{n \leq x} \frac{d_{1 / 2}(n)}{n^{s+i v}}\right|^{2} d t \ll T(\log T)^{9 / 4},
$$

where $d_{1 / 2}(n)$ are the coefficients of the Dirichlet series expansion

$$
\zeta(s)^{\frac{1}{2}}=\sum_{n \geq 1} \frac{d_{1 / 2}(n)}{n^{s}}, \quad \Re(s)>1 .
$$

Proof. Let

$$
\Phi_{x, v}(s):=\zeta(s) \cdot \sum_{n \leq x} \frac{d_{1 / 2}(n)}{n^{s+i v}}
$$

and

$$
f_{t}(s)=\frac{s-1}{s-3} \exp \left((s-i t)^{2}\right) .
$$

Then, by Gabriel's convexity theorem (see [HB81], Lemma 3)

$$
\begin{aligned}
\int_{\mathbb{R}}\left|\Phi_{x, v}(\sigma+i u) f_{t}(\sigma+i u)\right|^{2} d u & \leq\left(\int_{\mathbb{R}}\left|\Phi_{x, v}\left(\frac{1}{2}+i u\right) f_{t}\left(\frac{1}{2}+i u\right)\right|^{2} d u\right)^{\frac{5 / 2-\sigma}{2}} \\
& \times\left(\int_{\mathbb{R}}\left|\Phi_{x, v}\left(\frac{5}{2}+i u\right) f_{t}\left(\frac{5}{2}+i u\right)\right|^{2} d u\right)^{\frac{\sigma-1 / 2}{2}}
\end{aligned}
$$

We now integrate both sides over $T \leq t \leq 2 T$ and use Hölder's inequality to get

$$
\begin{aligned}
\int_{\mathbb{R}}\left|\Phi_{x, v}(\sigma+i u)\right|^{2} \widetilde{f}_{T}(\sigma+i u) d u & \leq\left(\int_{\mathbb{R}}\left|\Phi_{x, v}\left(\frac{1}{2}+i u\right)\right|^{2} \widetilde{f}_{T}\left(\frac{1}{2}+i u\right) d u\right)^{\frac{5 / 2-\sigma}{2}} \\
& \times\left(\int_{\mathbb{R}}\left|\Phi_{x, v}\left(\frac{5}{2}+i u\right)\right|^{2} \widetilde{f}_{T}\left(\frac{5}{2}+i u\right) d u\right)^{\frac{\sigma-1 / 2}{2}}
\end{aligned}
$$

where

$$
\widetilde{f}_{T}(\sigma+i u):=\int_{T}^{2 T}\left|f_{t}(\sigma+i u)\right|^{2} d t .
$$

Clearly $\widetilde{f}_{T}(\sigma+i u) \asymp 1$ if $T \leq u \leq 2 T$. In addition

$$
\tilde{f}_{T}(\sigma+i u) \ll \begin{cases}1 & \text { if } T / 2 \leq u \leq 3 T \\ e^{-|u|} & \text { otherwise. }\end{cases}
$$


We also note that $\Phi_{x, v}(s) \ll(1+|s|)^{1 / 4+\varepsilon} \cdot \sqrt{T}$. Therefore the previous inequality becomes

$$
\begin{aligned}
\int_{T}^{2 T}\left|\Phi_{x, v}(\sigma+i t)\right|^{2} d t \ll & \left(\int_{T / 2}^{3 T}\left|\Phi_{x, v}\left(\frac{1}{2}+i t\right)\right|^{2} d t+O(T)\right)^{\frac{5 / 2-\sigma}{2}} \\
& \times\left(\int_{T / 2}^{3 T}\left|\Phi_{x, v}(5 / 2+i t)\right|^{2} d t+O(T)\right)^{\frac{\sigma-1 / 2}{2}} .
\end{aligned}
$$

According to Theorem 1 the first integral on the right-hand side is $O\left(T(\log T)^{9 / 4}\right)$ while the second integral on the right hand side is $O(T)$.

Let $\delta>0$ be a small positive real number to be chosen later. We pick a parameter $\theta$ close to 1 , with $\delta<\theta<1$, and define

$$
\widehat{F}(z)=e^{2 \pi i(\theta-\delta) z} \cdot\left(\frac{e^{2 \pi i(1-\theta) z}-1}{2 \pi i(1-\theta) z}\right)^{N}
$$

with some bounded $N>10$. We see that $F$ is compactly supported on $[\theta-\delta, \theta-\delta+$ $(1-\theta) N]$. Define for $x>0$,

$$
G(x)=1-\int_{0}^{x} F(u) d u
$$

and $G(x)=0$ for $x \leq-1$. Moreover, we let $G(x)$ decay smoothly until 0 on the interval $[-1,0]$. This way $F=-G^{\prime}$ for $x>0$. We notice that $G(x)=1$ for $0<x<\theta-\delta$ and that $G(x)=1-\widehat{F}(0)=0$ for $x>\theta-\delta+(1-\theta) N$. Finally we notice that $G$ is $N$ times differentiable, and consequently that $\widehat{G}(x) \ll(1+|x|)^{-N}$.

Now we make a choice for $\theta$ and $\delta$. Let $\theta=\log y / \log x$ with $y=T^{1 / 2+2 \delta}$ and $x$ chosen so that $\theta-\delta+(1-\theta) N<1$. We pick $1-\theta=(\delta / 2) /(N-1)$ so that $x=y^{1 /(1-(\delta / 2) /(N-1))}$. Then, we choose $\delta$ small enough but positive so as to ensure that $x<T^{1 / 2+0.01}$.

Note that

$$
\widehat{F}\left(-\frac{i w \log x}{2 \pi}\right)=\left(y x^{-\delta}\right)^{w} \cdot\left(\frac{(x / y)^{w}-1}{w(1-\theta) \log x}\right)^{N}
$$

Let $s=\sigma+i t$ with $t \asymp T$ and $\sigma=\frac{1}{2}+\frac{A}{\log T}$, with $A>0$. Using Lemma 2 and shifting contours to $\Re(w)=\frac{1}{2}-\sigma$ we get

$$
\zeta(s)=\sum_{n} \frac{G\left(\frac{\log n}{\log x}\right)}{n^{s}}+\kappa(4 N)^{N} \frac{\left(y x^{-\delta}\right)^{1 / 2-\sigma}}{(\delta \log x)^{N}} \int_{-\infty}^{\infty} \frac{\left|\zeta\left(\frac{1}{2}+i t+i v\right)\right| d v}{\left(\left(\sigma-\frac{1}{2}\right)^{2}+v^{2}\right)^{(N+1) / 2}}+O\left(T^{-1}\right)
$$

where $O(1 / T)$ is the contribution from the pole at $w=1-s$ and with $|\kappa| \leq 1$. Let $c(m)=\sum_{m=f e, f, e \leq x} d_{1 / 2}(e) d_{1 / 2}(f)$. Importantly, notice that $c(m)=1$ for $m \leq x$. Since 
in addition $G(v)=0$ for $v>1$ we get

$$
\begin{aligned}
\sum_{n} \frac{G\left(\frac{\log n}{\log x}\right)}{n^{s}} & =\sum_{n} c(n) \frac{G\left(\frac{\log n}{\log x}\right)}{n^{s}} \\
& =\frac{\log x}{2 \pi} \int_{\mathbb{R}} \sum_{n} \frac{c(n)}{n^{s+i v}} \cdot \widehat{G}\left(\frac{v \log x}{2 \pi}\right) d v \\
& =\frac{\log x}{2 \pi} \int_{\mathbb{R}}\left(\sum_{n \leq x} \frac{d_{1 / 2}(n)}{n^{s+i v}}\right)^{2} \widehat{G}\left(\frac{v \log x}{2 \pi}\right) d v .
\end{aligned}
$$

Combining the above two equations, we have obtained the following inequality

$$
\begin{aligned}
|\zeta(s)| & \leq \log x \int_{\mathbb{R}}\left|\sum_{n \leq x} \frac{d_{1 / 2}(n)}{n^{s+i v}}\right|^{2} \cdot\left|\widehat{G}\left(\frac{v \log x}{2 \pi}\right)\right| d v \\
& +(4 N)^{N} \frac{\left(y x^{-\delta}\right)^{1 / 2-\sigma}}{(\delta \log x)^{N}} \int_{-\infty}^{\infty} \frac{\left|\zeta\left(\frac{1}{2}+i t+i v\right)\right| d v}{\left(\left(\sigma-\frac{1}{2}\right)^{2}+v^{2}\right)^{(N+1) / 2}}+O(1 / T) .
\end{aligned}
$$

Therefore we have obtained

$$
\int_{T}^{2 T}|\zeta(s)|^{3} d t \leqslant \log x \int_{\mathbb{R}}\left|\widehat{G}\left(\frac{v \log x}{2 \pi}\right)\right| \int_{T}^{2 T}|\zeta(s)|^{2} \cdot\left|\sum_{n \leq x} \frac{d_{1 / 2}(n)}{n^{s+i v}}\right|^{2} d t d v+\mathcal{E}+O\left(T^{\varepsilon}\right),
$$

where

$\mathcal{E} \leqslant(4 N)^{N} \cdot \frac{\left(y x^{-\delta}\right)^{1 / 2-\sigma}}{(\delta \log x)^{N}} \int_{-\infty}^{\infty}\left(\int_{T}^{2 T}|\zeta(s)|^{2} \cdot\left|\zeta\left(\frac{1}{2}+i t+i v\right)\right| d t\right) \cdot \frac{d v}{\left(\left(\sigma-\frac{1}{2}\right)^{2}+v^{2}\right)^{(N+1) / 2}}$.

By Hölder's inequality and the bound $\left|\zeta\left(\frac{1}{2}+i t\right)\right| \ll(1+|t|)^{1 / 6+\varepsilon}$, for $|v|<T^{1 / 100}$ we have

$$
\begin{aligned}
\int_{T}^{2 T}|\zeta(s)|^{2}\left|\zeta\left(\frac{1}{2}+i t+i v\right)\right| d t & \leqslant\left(\int_{T}^{2 T}|\zeta(s)|^{3} d t\right)^{2 / 3}\left(\int_{T}^{2 T}|\zeta(1 / 2+i t+i v)|^{3} d t\right)^{1 / 3} \\
& \leqslant M_{3}(\sigma, T)^{2 / 3} \cdot\left(M_{3}\left(\frac{1}{2}, T\right)+O\left(|v| T^{1 / 2+\varepsilon}\right)\right)^{1 / 3}
\end{aligned}
$$

where

$$
M_{3}(\sigma, T):=\int_{T}^{2 T}|\zeta(\sigma+i t)|^{3} d t
$$

By a minor modification of Lemma 4 in Heath-Brown's paper [HB81] we have

$$
M_{3}\left(\frac{1}{2}, T\right) \leqslant C \cdot T^{(3 / 2)(\sigma-1 / 2)} M_{3}(\sigma, T) .
$$

Therefore,

$$
\int_{T}^{2 T}|\zeta(s)|^{2}\left|\zeta\left(\frac{1}{2}+i t+i v\right)\right| d t \leq M_{3}(\sigma, T)^{2 / 3} \cdot\left(C T^{(3 / 2)\left(\sigma-\frac{1}{2}\right)} M_{3}(\sigma, T)+O\left(|v| T^{1 / 2+\varepsilon}\right)\right)^{1 / 3}
$$




$$
\leq C T^{(1 / 2)\left(\sigma-\frac{1}{2}\right)} \cdot\left(M_{3}(\sigma, T)+O\left(T^{1-\varepsilon}\right)\right) .
$$

The contribution of $|v|>T^{1 / 100}$ to (6.2) is negligible, provided that $N$ is chosen to be large enough. We conclude that

$$
\mathcal{E} \leqslant \frac{C(4 N)^{N}}{\delta^{N}}\left(\frac{T^{1 / 2} x^{\delta}}{y}\right)^{\sigma-\frac{1}{2}} \cdot \frac{M_{3}(\sigma, T)}{\left(\log x\left(\sigma-\frac{1}{2}\right)\right)^{N}}+O_{A}\left(T^{1-\varepsilon}\right) .
$$

Using Lemma 3, we find that

$$
\log x \int_{\mathbb{R}}\left|\widehat{G}\left(\frac{v \log x}{2 \pi}\right)\right| \int_{T}^{2 T}|\zeta(s)|^{2} \cdot\left|\sum_{n \leq x} \frac{d_{1 / 2}(n)}{n^{s+i v}}\right|^{2} d t d v \ll T(\log T)^{9 / 4} .
$$

We have obtained the inequality

$$
M_{3}(\sigma, T) \ll T(\log T)^{9 / 4}+O_{A}\left(T^{1-\varepsilon}\right)+\frac{C(4 N)^{N}}{\delta^{N}} \cdot\left(\frac{T^{1 / 2} x^{\delta}}{y}\right)^{\sigma-\frac{1}{2}} \cdot \frac{M_{3}(\sigma, T)}{\left(\left(\sigma-\frac{1}{2}\right) \log x\right)^{N}} .
$$

Recall that $y=T^{1 / 2+2 \delta}<x<T$. Since $\sigma=\frac{1}{2}+\frac{A}{\log T}$, the third term on the right-hand side in the above equation is less than

$$
\leq C(8 N / A)^{N} \delta^{-N} e^{-\delta A} M_{3}(\sigma, T)
$$

with $C$ an absolute constant. Thus, if $A$ is large enough (but bounded) then the third term on the right-hand side in the above equation is absorbed into the left-hand side, and we conclude that

$$
M_{3}(\sigma, T) \ll T(\log T)^{9 / 4} .
$$

Since $M_{3}\left(\frac{1}{2}, T\right) \ll T^{(3 / 2) \cdot\left(\sigma-\frac{1}{2}\right)} M_{3}(\sigma, T)$ by Lemma 4 of Heath-Brown [HB81] and since $\sigma=\frac{1}{2}+\frac{A}{\log T}$, we obtain that

$$
M_{3}\left(\frac{1}{2}, T\right) \ll T(\log T)^{9 / 4}
$$

6.1. Moments of the form $k=1+1 / n$. Since we do not claim the result for moments with $k=1+1 / n$ we only sketch the necessary modifications of the previous argument, for the convenience of the interested reader. In order to adapt our argument above to moments of the form $1+1 / n$, it suffices to prove the inequality

$$
\begin{aligned}
|\zeta(s)|^{2 / n} & \ll \log T \int_{\sigma_{-}}^{\sigma_{+}} \int_{\mathbb{R}}\left|\hat{G}\left(\frac{v}{2 \pi}\right)\right|^{2 / n} \cdot \mid \sum_{m \leqslant x} \frac{d_{1 / n}(m)}{\left.m^{s+\sigma+i v / \log x}\right|^{2} d v d \sigma} \\
& +\frac{\left(y x^{-\delta}\right)^{2(1 / 2-\sigma) / n}}{(\delta \log x)^{2 N / n}} \cdot \log T \int_{\sigma_{-}}^{\sigma_{+}} \int_{-\infty}^{\infty} \frac{|\zeta(\sigma+i t+i v)|^{2 / n} \cdot d v d \sigma}{\left(\left(\sigma-\frac{1}{2}\right)^{2}+v^{2}\right)^{(N+1) / n}}
\end{aligned}
$$

where $\sigma_{-}=\frac{1}{2}-\frac{1}{\log T}, \sigma_{+}=\frac{1}{2}+\frac{1}{\log T}$ and with the implicit constant depending at most on $n, N$ and the same choice of parameters $\theta, x, y, \delta$. This is sufficient because the previous 
argument does not depend on some specific quantification of the dependence on $N$. First we note that

$$
\sum_{n} \frac{G\left(\frac{\log n}{\log x}\right)}{n^{s}}=\frac{1}{2 \pi} \int_{\mathbb{R}}\left(\sum_{m \leqslant x} \frac{d_{1 / n}(m)}{m^{s+i v / \log x}}\right)^{n} \cdot \hat{G}\left(\frac{v}{2 \pi}\right) d v .
$$

Combining this with Lemma 2, and using the same choice of parameters $\theta, x, y, \delta$ as before, it follows that

$$
|\zeta(s)| \ll \int_{\mathbb{R}}\left|\hat{G}\left(\frac{v}{2 \pi}\right)\right| \cdot\left|\sum_{m \leqslant x} \frac{d_{1 / n}(m)}{m^{s+\sigma+i v / \log x}}\right|^{n} d v+\frac{\left(y x^{-\delta}\right)^{1 / 2-\sigma}}{(\delta \log x)^{N}} \int_{\mathbb{R}} \frac{\left|\zeta\left(\frac{1}{2}+i t+i v\right)\right| d v}{\left(\left(\sigma-\frac{1}{2}\right)^{2}+v^{2}\right)^{(N+1) / 2}} .
$$

Taking the $2 / n$ power on both sides, it remains to show that

$$
\left(\int_{\mathbb{R}}\left|\hat{G}\left(\frac{v}{2 \pi}\right)\right| \cdot\left|\sum_{m \leqslant x} \frac{d_{1 / n}(m)}{m^{s+i v / \log x}}\right|^{n} d v\right)^{\frac{2}{n}} \ll \log T \int_{\sigma_{-}}^{\sigma_{+}} \int_{\mathbb{R}}\left|\hat{G}\left(\frac{v}{2 \pi}\right)\right|^{\frac{2}{n}} \cdot\left|\sum_{m \leqslant x} \frac{d_{1 / n}(m)}{m^{s+\sigma+i v / \log x}}\right|^{2} d v d \sigma,
$$

and that

$$
\left(\int_{-\infty}^{\infty} \frac{\left|\zeta\left(\frac{1}{2}+i t+i v\right)\right| d v}{\left(\left(\sigma-\frac{1}{2}\right)^{2}+v^{2}\right)^{(N+1) / 2}}\right)^{2 / n} \ll \log T \int_{\sigma_{-}}^{\sigma_{+}} \int_{-\infty}^{\infty} \frac{|\zeta(\sigma+i t+i v)|^{2 / n} d v}{\left(\left(\sigma-\frac{1}{2}\right)^{2}+v^{2}\right)^{(N+1) / n}} d \sigma
$$

We will only show how to prove the second inequality since the proof of the first is very similar. We bound the integral

$$
\int_{-\infty}^{\infty} \frac{\left|\zeta\left(\frac{1}{2}+i t+i v\right)\right| d v}{\left(\left(\sigma-\frac{1}{2}\right)^{2}+v^{2}\right)^{(N+1) / 2}} \leq\left(\sigma-\frac{1}{2}\right)^{-N-1} \sum_{k} \frac{M_{k}}{(1+|k|)^{N+1}}
$$

where $M_{k}$ is the maximum of $\zeta\left(\frac{1}{2}+i t+i v\right)$ over the interval $\left|v-k\left(\sigma-\frac{1}{2}\right)\right|<\left(\sigma-\frac{1}{2}\right) / 2$. Therefore

$$
\left(\int_{-\infty}^{\infty} \frac{\left|\zeta\left(\frac{1}{2}+i t+i v\right)\right| d v}{\left(\left(\sigma-\frac{1}{2}\right)^{2}+v^{2}\right)^{(N+1) / 2}}\right)^{2 / n} \leq\left(\sigma-\frac{1}{2}\right)^{-2(N+1) / n} \sum_{k} \frac{M_{k}^{2 / n}}{(1+|k|)^{2(N+1) / n}}
$$

By sub-harmonicity,

$$
M_{k}^{2 / n} \ll \log T \int_{\sigma_{-}}^{\sigma_{+}} \int_{(k-1)\left(\sigma-\frac{1}{2}\right)}^{(k+1)\left(\sigma-\frac{1}{2}\right)}|\zeta(\sigma+i t+i x)|^{2 / n} d x d \sigma .
$$

We conclude that

$$
\begin{aligned}
& \left(\sigma-\frac{1}{2}\right)^{-2(N+1) / n} \sum_{k} \frac{M_{k}^{2 / n}}{(1+|k|)^{2(N+1) / n}} \\
& \ll \log T \int_{\sigma_{-}}^{\sigma_{+}} \sum_{k} \int_{(k-1)\left(\sigma-\frac{1}{2}\right)}^{(k+1)\left(\sigma-\frac{1}{2}\right)} \frac{|\zeta(\sigma+i t+i x)|^{2 / n} d x d \sigma}{\left(\left(\sigma-\frac{1}{2}\right)^{2}+\left(k\left(\sigma-\frac{1}{2}\right)\right)^{2}\right)^{2(N+1) / n}} \\
& \ll \log T \int_{\sigma_{-}}^{\sigma_{+}} \int_{-\infty}^{\infty} \frac{|\zeta(\sigma+i t+i v)|^{2 / n} d v}{\left(\left(\sigma-\frac{1}{2}\right)^{2}+v^{2}\right)^{(N+1) / n}} d \sigma .
\end{aligned}
$$


Combining these equations together, we obtain the desired inequality (6.3).

\section{Appendix A. On Conjecture 1}

Proposition 4. Let $A, M, N \geq 1$ and let $A \ll(M N)^{\frac{1}{2}+\varepsilon}$. Then

$$
\max _{\alpha, \beta, \nu}\left|S_{A, M, N}\right| \gg(A M N)^{\frac{1}{2}-\varepsilon}(M+N)^{\frac{1}{2}}+A(M+N)^{1-\varepsilon},
$$

for all $\varepsilon>0$, where the maximum is taken over all choices of coefficients $\alpha_{m}, \beta_{n}, \nu_{a} \ll 1$.

Proof. By the reciprocity relation $\frac{\bar{m}}{n} \equiv-\frac{\bar{n}}{m}+\frac{1}{m n}(\bmod 1)$ we can assume $M \geq N$. Moreover, we can assume $N, A \gg M^{\varepsilon}$ for some small $\varepsilon>0$ and $M$ arbitrary large, since otherwise the result is easy.

First, we consider the case $M^{1-\delta} \gg N$ for some $\delta>0$ and we take $\alpha_{m}=f(m)$ for some smooth function $f:[M, 2 M] \rightarrow[0,1]$ which is such that $f^{(j)}(x) \ll_{j} x^{-j}$ for all $j \geq 0$ and $\int_{\mathbb{R}} f(x)=K M$, for some $K>0$. Also, let $\beta_{n}=-\gamma_{n}$, where $\gamma_{n}$ is the indicator function of the primes congruent to $1(\bmod 4)$ in $[N, 2 N]$, and let $\nu_{a}$ be the indicator function of the primes congruent to $3(\bmod 4)$ in $[A, 2 A]$.

By Poisson summation, we have

$$
\sum_{m} f(m) \mathrm{e}\left(\frac{a \bar{m}}{n}\right)=K \frac{M}{N}\left(c_{n}(a)+O\left(M^{-100}\right)\right),
$$

where

$$
c_{n}(a)=\sum_{\substack{b=1,(b, n)=1}}^{n} \mathrm{e}\left(\frac{b a}{n}\right)=\mu\left(\frac{n}{(n, a)}\right) \frac{\varphi(n)}{\varphi\left(\frac{n}{(n, a)}\right)}
$$

is the Ramanujan sum. It follows that

$$
\begin{aligned}
S_{A, M, N} & =K \frac{M}{N} \sum_{a} \sum_{n} \beta_{n} \nu_{a}\left(c_{n}(a)+O\left(M^{-100}\right)\right) \\
& =K \frac{M}{N} \sum_{a} \sum_{n} \gamma_{n} \nu_{a}\left(1+O\left(M^{-100}\right)\right) \gg(M A)^{1-\varepsilon} .
\end{aligned}
$$

We now prove

$$
\max _{\alpha, \beta, \nu}\left|S_{A, M, N}\right| \gg M(A N)^{\frac{1}{2}-\varepsilon},
$$

which then implies the Proposition even in the case $M^{1-\delta} \ll N$ for all $\delta>0$.

By choosing $\alpha_{m}$ appropriately, we have

$$
\max _{\alpha, \beta, \nu}\left|S_{A, M, N}\right| \gg \max _{\beta, \nu} \sum_{m} F_{m ; \beta, \nu}
$$

where

$$
F_{m ; \beta, \nu}:=\left|\sum_{a} \sum_{(n, m)=1} \nu_{a} \beta_{n} \mathrm{e}\left(\frac{a \bar{m}}{n}\right)\right| .
$$


First, notice that we have

$$
\max _{\beta, \nu} \sum_{m} F_{m ; \beta, \nu} \geq \frac{1}{\varphi(q)^{2}} \sum_{\chi_{1}, \chi_{2}} \sum_{(\bmod q)} F_{m ; \beta\left(\chi_{1}\right), \nu\left(\chi_{2}\right)}
$$

with $q$ any prime greater than $4\left(A^{4}+N^{4}\right)$ and where $\beta\left(\chi_{1}\right), \nu\left(\chi_{2}\right)$ denotes sequences defined by $\beta\left(\chi_{1}\right)_{n}=\chi_{1}(n)$ and $\nu\left(\chi_{2}\right)_{a}=\chi_{2}(a)$ respectively. Moreover, by Hölder's inequality,

$$
\begin{aligned}
\frac{1}{\varphi(q)^{2}} & \sum_{\chi_{1}, \chi_{2}(\bmod q)} \sum_{m} F_{m ; \beta\left(\chi_{1}\right), \nu\left(\chi_{2}\right)}^{2} \leq\left(\frac{1}{\varphi(q)^{2}} \sum_{\chi_{1}, \chi_{2}(\bmod q)} \sum_{m} F_{m ; \beta\left(\chi_{1}\right), \nu\left(\chi_{2}\right)}\right)^{\frac{2}{3}} \times \\
& \times\left(\frac{1}{\varphi(q)^{2}} \sum_{\chi_{1}, \chi_{2}(\bmod q)} \sum_{m} F_{m ; \beta\left(\chi_{1}\right), \nu\left(\chi_{2}\right)}^{4}\right)^{\frac{1}{3}} .
\end{aligned}
$$

The left hand side is

$$
\frac{1}{\varphi(q)^{2}} \sum_{\chi_{1}, \chi_{2}(\bmod q)} \sum_{m} F_{m ; \beta\left(\chi_{1}\right), \nu\left(\chi_{2}\right)}^{2}=\sum_{m} \sum_{a} \sum_{(n, m)=1} 1 \gg M A N
$$

and we also have

$$
\frac{1}{\varphi(q)^{2}} \sum_{\chi_{1}, \chi_{2}(\bmod q)} \sum_{m} F_{m ; \beta\left(\chi_{1}\right), \nu\left(\chi_{2}\right)}^{4}=\sum_{m} \sum_{a_{1} a_{2}=a_{3} a_{4}} \sum_{\substack{n_{1} n_{2}=n_{3} n_{4},\left(m, n_{1} n_{2}\right)=1}} 1 \ll M(A N)^{2+\varepsilon} .
$$

Thus,

$$
\frac{1}{\varphi(q)^{2}} \sum_{\chi_{1}, \chi_{2}(\bmod q)} F_{m ; \beta\left(\chi_{1}\right), \nu\left(\chi_{2}\right)} \gg M(A N)^{\frac{1}{2}-\varepsilon}
$$

and the proposition follows.

\section{REFERENCES}

[BC] S. Bettin and V. Chandee, Trilinear forms with kloosterman fractions, preprint.

[BCHB85] R. Balasubramanian, J. B. Conrey, and D. R. Heath-Brown, Asymptotic mean square of the product of the Riemann zeta-function and a Dirichlet polynomial, J. Reine Angew. Math. 357 (1985), 161-181. MR 783539 (87f:11061)

[Bre] J. Bredberg, Large gaps between consecutive zeros, on the critical line, of the Riemann zeta-function, arXiv: 1101.3197.

[CGG86] J. B. Conrey, A. Ghosh, and S. M. Gonek, Large gaps between zeros of the zeta-function, Mathematika 33 (1986), no. 2, 212-238 (1987). MR 882495 (88g:11057)

[Con89] J. B. Conrey, More than two fifths of the zeros of the Riemann zeta function are on the critical line, J. Reine Angew. Math. 399 (1989), 1-26. MR 1004130 (90g:11120)

[Dav00] Harold Davenport, Multiplicative number theory, third ed., Graduate Texts in Mathematics, vol. 74, Springer-Verlag, New York, 2000, Revised and with a preface by Hugh L. Montgomery. MR 1790423 (2001f:11001)

[DFI97a] W. Duke, J. Friedlander, and H. Iwaniec, Bilinear forms with Kloosterman fractions, Invent. Math. 128 (1997), no. 1, 23-43. MR 1437494 (97m:11109) 
[DFI97b] _ Representations by the determinant and mean values of L-functions, Sieve methods, exponential sums, and their applications in number theory (Cardiff, 1995), London Math. Soc. Lecture Note Ser., vol. 237, Cambridge Univ. Press, Cambridge, 1997, pp. 109-115. MR 1635738 (99f:11105)

[DI84] J.-M. Deshouillers and H. Iwaniec, Power mean-values for Dirichlet's polynomials and the Riemann zeta-function. II, Acta Arith. 43 (1984), no. 3, 305-312. MR 738142 (85g:11076)

[DI83] Kloosterman sums and Fourier coefficients of cusp forms, Invent. Math. 70 (1982/83), no. 2, 219-288. MR 684172 (84m:10015)

[GR07] I. S. Gradshteyn and I. M. Ryzhik, Table of integrals, series, and products, seventh ed., Elsevier/Academic Press, Amsterdam, 2007, Translated from the Russian, Translation edited and with a preface by Alan Jeffrey and Daniel Zwillinger, With one CD-ROM (Windows, Macintosh and UNIX). MR 2360010 (2008g:00005)

[Har] A. Harper, Sharp conditional bounds for moments of the riemann zeta function, arXiv:1305.4618.

[HB81] D. R. Heath-Brown, Fractional moments of the Riemann zeta function, J. London Math. Soc. (2) 24 (1981), no. 1, 65-78. MR 623671 (82h:10052)

[HY10] C. P. Hughes and Matthew P. Young, The twisted fourth moment of the Riemann zeta function, J. Reine Angew. Math. 641 (2010), 203-236. MR 2643931 (2011h:11092)

[LR] X. Li and M. Radziwilt, The Riemann-zeta function on vertical arithmetic progressions, Int. Math. Res. Not. IMRN, to appear, arXiv: 1208.2684.

[Rad] Maksym Radziwill, Limitations to mollifying $\zeta(s)$, arXiv:1207.6583.

[Rad12] _ The 4.36th moment of the Riemann zeta-function, Int. Math. Res. Not. IMRN (2012), no. 18, 4245-4259. MR 2975381

[Sou95] K. Soundararajan, Mean-values of the Riemann zeta-function, Mathematika 42 (1995), no. 1, 158-174. MR 1346680 (96h:11086)

[Wat95] N. Watt, Kloosterman sums and a mean value for Dirichlet polynomials, J. Number Theory 53 (1995), no. 1, 179-210. MR 1344840 (96f:11109)

Centre de Recherches Mathematiques Universite de Montreal P. O Box 6128, Centre-

Ville Station Montreal, Quebec H3C 3J7

E-mail address: bettin@crm.umontreal.ca

Department of Mathematics, Burapha University, 169 Long-hard bangsaen rd SaenSuk, Mueang, Chonburi, Thailand 20131

E-mail address: vorrapan@buu.ac.th

School of Mathematics, Institute for Advanced Study, 1 Einstein Drive, Princeton, NJ, 08540

E-mail address: maksym@ias.edu

Current address: Centre de Recherches Mathematiques Universite de Montreal P. O Box 6128, Centre-Ville Station Montreal, Quebec H3C 3J7 